\title{
SOME RECENT PROGRESS IN SINGULAR STOCHASTIC PARTIAL DIFFERENTIAL EQUATIONS
}

\author{
IVAN CORWIN AND HAO SHEN
}

\begin{abstract}
Stochastic partial differential equations are ubiquitous in mathematical modeling. Yet, many such equations are too singular to admit classical treatment. In this article we review some recent progress in defining, approximating, and studying the properties of a few examples of such equations. We focus mainly on the dynamical $\Phi^{4}$ equation, the KPZ equation, and the parabolic Anderson model, as well as a few other equations which arise mainly in physics.
\end{abstract}

\section{Contents}

1. Introduction

2. A first (linear) SPDE 410

3. Nonlinear SPDEs 415

4. Well-posedness of singular SPDEs

5. Renormalization in physical models 431

6. Singular SPDEs as universal objects $\quad 438$

Acknowledgments

About the authors

References 447

\section{INTRODUCTION}

Partial differential equations (PDEs) and randomness are ubiquitous constructions used to model both mathematical and physical phenomena. For instance, PDEs have been used for centuries to describe the building block laws of physics, and to model aggregate macroscopic phenomena, such as heat conduction, diffusion, electro-magnetic dynamics, interface and fluid dynamics. Randomness has become a default paradigm for modeling systems with uncertainty or with many complicated or chaotic microscopic interactions.

Combining these two approaches leads to the study of stochastic PDEs (SPDEs) in which the coefficients or forcing terms in PDEs are described via certain random processes. While SPDEs have become increasingly important in applications, there remain many fundamental mathematical challenges in their study - in particular,

Received by the editors April 8, 2019.

The first author was partially supported by the Packard Fellowship for Science and Engineering, and by the NSF through DMS-1811143 and DMS-1664650.

The second author was partially supported by the NSF through DMS-1712684 and DMS1909525. 
showing how they arise from microscopic particle based models remains a major source of research problems and has seen some radical progress in the past decade.

The purpose of this article is to introduce a few important classes of SPDEs and to describe how they arise and the mathematical challenges that go along with demonstrating that. Though this article will mainly focus on nonlinear systems, we will start our investigation in Section 2 in the simpler and more classical setting of linear SPDEs which are very well understood. In Section 3 we turn our attention to nonlinear SPDEs and introduce our two main examples (the dynamical $\Phi^{4}$ equation and the KPZ (Kardar-Parisi-Zhang) equation) along with a host of other important SPDEs which arise in physics. Our discussion in this section is heuristic and ignores some of the serious mathematical challenges which arise when one tries to make sense of what it means to "solve" an SPDE. This challenge is addressed in Section 4 . In the course of making sense of SPDEs, there are often renormalizations which arise (effectively changing the equation). Section 5 describes how these renormalizations have physical meaning and arise in certain discrete approximation schemes for the continuum equations. Finally, Section 6 seeks to demonstrate how these SPDEs (in particular, the KPZ equation) arise as universal limits from microscopic systems.

Before proceeding to our main text, one disclaimer. Our aim is to make this material approachable to nonexperts. As such, we will not state precise theorems or give proofs but rather will attempt to provide some intuition behind results and the challenges which accompany proving them. An interested reader can find much more detail and precision in the works cited or, can consult other survey articles such as GP18b, Gub18, CW17, Hai15a, Hai14a].

\section{A first (LINEAR) SPDE}

We will start our discussion on linear SPDEs with the stochastic heat equation which is driven by a random additive noise term $\xi$ :

$$
\partial_{t} u(t, x)=\partial_{x}^{2} u(t, x)+\xi(t, x),
$$

where $\xi$ is the so-called space-time white noise. It will take a bit of work to define this noise and make sense of what it means to solve this equation. However, before going down that route, we will first address the question of what sort of physical system does this model? In particular, we will explain heuristically how this equation arises from a simple microscopic model of polymers in liquid.

Consider modeling a polymer chain (e.g., composed of DNA or proteins) in a liquid. A simple model involves describing the polymer by a string of $N$ beads that are linked together sequentially by springs and that are subject to kicking by noise, as shown in Figure 2.1, where $N=13$ : Imagine that each bead of the polymer is kicked by the surrounding liquid molecules. In our simplified model 11 we describe such a system via the following equations of motion for the position $r_{i} \in \mathbf{R}^{3}$ of the $i$ th bead:

$$
d r_{i}(t)=\left(r_{i+1}(t)-r_{i}(t)\right) d t+\left(r_{i-1}(t)-r_{i}(t)\right) d t+w_{i}(t) \quad\left(t \in \mathbf{R}_{+}, i=1, \ldots, N\right)
$$

\footnotetext{
${ }^{1}$ As usual, one always has to make various simplifying assumptions in order to describe a complicated physical system via a mathematically analyzable model. It is natural to ask whether having random kicking leads to a reasonable microscopic model. After all, the liquid itself is governed by certain physical laws of motion for its particles. Such concerns arose early in the development of Brownian motion as the model for a single tracer particle moving in a liquid; see Bru68 for a nice historical review. We do not provide further justification for this as a reasonable microscopic model here.
} 


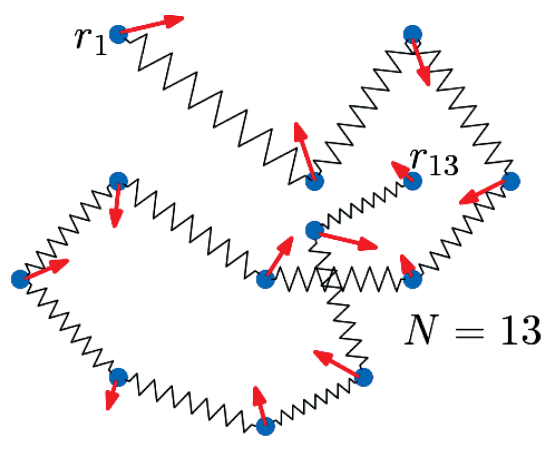

Figure 2.1. The dots represent the locations of the beads connected by zigzag edges. The arrows represent the forces acting to move the beads - partly due to spring force with the previous and subsequent beads, and part due to some random kicking force (yet to be specified).

where $w_{i}(t)$ is random kicking at time $t$, and where a boundary condition is given by fixing $r_{0}-r_{1} \equiv 0$ and $r_{N+1}-r_{N} \equiv 0$ for all time. Equation (2.2) means the following.

- The linear drift terms $\left(r_{i+1}-r_{i}\right) d t$ and $\left(r_{i-1}-r_{i}\right) d t$ arise from assuming a linear spring force between the $i$ th bead with its neighboring (in the sense of label number) beads. Without the kicking term $w_{i}(t)$, equation (2.2) would simply be a coupled system of $N$ ordinary differential equations.

- The term $w_{i}(t)$ represents the random kicking that is experienced by the $i$ th bead at time $t$. We make the simplifying assumption that the kicking is "overdamped" 2 and we model the kicks in terms of random jumps in the location of the $r_{i}(t)$. Namely, for each particle $r_{i}$ there is a random sequence of kicking times $3 t_{i, 1}<t_{i, 2}<\cdots$. At the kicking time $t_{i, j}$, we update $r_{i} \mapsto r_{i}+w_{i}\left(t_{i, j}\right)$, where $w_{i}\left(t_{i, j}\right)$ is an $\mathbf{R}^{3}$-valued random variable. We assume that the $w_{i}$ are statistically isotropic (i.e., their distribution is invariant under rotation) and are all independent and identically distributed (i.i.d.). Note that the resulting $r_{i}(t)$ process is piecewise continuous, with jumps occurring at the kicking times.

The question with which we are concerned is what happens to the polymer when its length grows, and possibly space and time are scaled accordingly. By default one might expect that as $N$ increases, the complexity of studying this system goes likewise. However, it turns out that there is a very tractable continuum limit for the evolution of our polymer model. That is to say, in the scaling limit, things simplify! In fact, this limit is quite robust and (up to some scaling constants) is not affected by various changes in the microscopic model, such as how we model the kicking (e.g., different distribution on the $w_{i}$ or on the kicking times). This robustness can, itself, be seen as evidence that the microscopic model may be reasonable.

\footnotetext{
${ }^{2}$ Essentially, this means that the kicks occur instantaneously in time and do not result in any inertia. This effectively decouples the various kicks.

${ }^{3}$ It is natural to assume the gaps between times $t_{i, j}-t_{i, j-1}$ are chosen according to independent exponential random variables of mean 1 . In this case, the times are distributed as a Poisson point process of intensity 1 .
} 
With the aim of demonstrating a continuum limit of our model, think of $N$ as large and define

$$
u_{N}(t, x):=\frac{1}{\sqrt{N}} r_{[x N]}\left(N^{2} t\right) \in \mathbf{R}^{3},
$$

where $x \in[0,1]$ encodes the label $i$ via $i=[x N]$ (the closest integer to $x N$ ). The linear drift term in (2.2) is in fact a discrete Laplacian, so under our diffusive scaling (that is, scaling $x$ by $N$ and $t$ by $N^{2}$ ) it approximates a continuum Laplacian $\partial_{x}^{2}$. Thus, for large $N$ one can expect that the following relation approximately holds:

$$
\partial_{t} u_{N}(t, x) \approx \partial_{x}^{2} u_{N}(t, x)+N^{\frac{3}{2}} w_{[N x]}\left(N^{2} t\right) .
$$

In the scaled coordinates, the kicking starts to add up. Namely, in a time-space region of size $d t \times d x$, there are roughly $N^{2} d t \times N d x$ kicks. By the central limit theorem the sum of $N^{2} \times N$ i.i.d. random variables divided by $N^{\frac{3}{2}}$ converges to a Gaussian random variable. Thus, on each time-space region, the kicking adds up to a Gaussian random variable with variance equal to the area of the region. Different regions have covariance given by the area of their overlap. This limit is called space-time (or time-space, given our ordering of variables) white noise and is denoted by $\xi(t, x)$. This heuristic leads us to the following typ 4 of limit as $N \rightarrow \infty$,

$$
u_{N}(t, x) \rightarrow u(t, x),
$$

where $\partial_{t} u(t, x)=\partial_{x}^{2} u(t, x)+\xi(t, x)$, and where $\xi(t, x)$ is space-time white noise.

Equation (2.5) is our first example of an SPDE - it is called the linear stochastic heat equation with additive noise. Even in this simple linear example we encounter an equation which requires some work to make sense of because of the noise. For convenience of our exposition, from this point forward, we will think of $x$ as a spatial variable (although in our example it actually stands for the parametrization of the limiting polymer length); and although $u$ and $\xi$ are $\mathbf{R}^{3}$-valued, the three components are completely independent (decoupled), so it will be convenient to simply consider equation (2.5) as $\mathbf{R}$-valued instead of $\mathbf{R}^{3}$-valued in the rest of this paper.

Let us look at equation (2.5) more closely, with spatial dimension $d$ now being arbitrary (recall $d=1$ corresponds with the above polymer example),

$$
\partial_{t} u(t, x)=\Delta u(t, x)+\xi(t, x), \quad x \in[0,1]^{d} \subset \mathbf{R}^{d},
$$

with, for instance, periodic boundary condition.

Consider the case $d=0$, where (2.6) becomes the stochastic (ordinary) differential equation $\partial_{t} u(t)=\xi(t)$. The $d=0$ white noise $\xi(t)$ is defined to be the derivative of Brownian motion so that $u(t)=\int_{0}^{t} \xi(s)$ is a Brownian motion. Of course, Brownian motion is famously almost nowhere differentiable, so $\xi$ is not defined as a function. Rather, $\xi(t)$ can be defined as a random distribution in a suitable negative regularity space (such as a negative Sobolev space). Since the Hölder regularity of Brownian motion is $1 / 2-$ (meaning any exponent below $1 / 2$ ), its derivative is said to have regularity $-1 / 2-$. There are other ways to define $\xi(t)$. For instance, if we restrict to a periodic $t \in[0, T]$, then $\xi(t)=\sum_{k \in \mathbf{Z}} N_{k} e_{k}(t)$,

\footnotetext{
${ }^{4}$ This type of convergence result was first proved by Funaki Fun83 in the slightly different setting where the $r_{i}$ are driven by Brownian motions. In the present setting, we do not know if a precise result of this sort has been proved (though we have no doubt that it can be). We are suppressing coefficients which may (depending on the nature of the discrete noise) arise in the limiting equation.
} 
where the $N_{k}$ are i.i.d. Gaussian random variables, and the $\left\{e_{k}\right\}_{k \in \mathbf{Z}}$ constitute an orthonormal basis of $L^{2}([0, T])$. Alternatively, one can define $\xi(t)$ via the machinery of Gaussian processes (see, for instance [Jan97]) wherein it suffices to specify its mean and variance. By definition, $\xi$ is mean zero, and since $\xi$ is a distribution as mentioned above, its covariance

$$
\mathbf{E}\left(\xi(t) \xi\left(t^{\prime}\right)\right)=\delta\left(t-t^{\prime}\right)
$$

(here $\mathbf{E}$ represents the expectation value operator and $\delta$ is a Dirac delta function) must be interpreted in a distributional sense as well. For a smooth test function $f$, one defines the stochastic integral $\xi(f)=\int f(t) \xi(t)$. Then $\xi$ is defined by the property that $\mathbf{E}(\xi(f))=0$ for all test functions $f$, and for test functions $f$ and $g$,

$$
\mathbf{E}(\xi(f) \xi(g))=\langle f, g\rangle_{L^{2}} .
$$

The random distribution $\xi$ is defined from this information using Kolmogorov's continuity theorem.

For general dimension $d \geq 1$, space-time white noise can be defined via analogous methods. As a Gaussian process, $\xi(t, x)$ is a random distribution with covariance

$$
\mathbf{E}\left(\xi(t, x) \xi\left(t^{\prime}, x^{\prime}\right)\right)=\delta\left(t-t^{\prime}\right) \delta\left(x-x^{\prime}\right),
$$

where the last $\delta$ is the Dirac delta function on $d$-dimensional space. Its action on space-time test functions $f$ and $g$ has covariance given by (2.7). where the $\langle\cdot, \cdot\rangle_{L^{2}}$ is $L^{2}$ product over space-time.

As the dimension $d$ increases, the regularity of $\xi$ decreases. We will work with spaces of space-time distributions (for $\alpha<0$ ) or functions (for $\alpha \geq 0$ ) denoted by $\mathcal{C}^{\alpha}$. These are essentially equivalent to the Besov spaces $\mathcal{B}_{\infty, \infty}^{\alpha}$ in harmonic analysis, and their precise definitions can be given via wavelets in [Hai14b, Eq. (3.2)]. The smaller $\alpha$ corresponds to less regular (or more singular) functions or distributions. A well-known result is that the space-time white noise $\xi \in \mathcal{C}^{\alpha}$ for $\alpha<-\frac{d+2}{2} 5$

Having made sense of $\xi$, it remains to understand what it means to solve equation (2.6). For a linear equation as (2.6), the meaning of solution is not hard to define; essentially one only needs to give a suitable meaning to the inverted linear differential operator acting on the noise $\xi$ : given an initial data $u(0, x)=u_{0}(x)$, the solution to (2.6) is defined by

$$
u=\left(\partial_{t}-\Delta\right)^{-1} \xi+e^{t \Delta} u_{0} .
$$

Here $e^{t \Delta}$ is the heat semigroup so that $e^{t \Delta} u_{0}(x):=\int_{[0,1]^{d}} P(t, x-y) u_{0}(y) d y$ solves the classical (deterministic) heat equation starting from $u_{0}$, with heat kernel $P(t, x):=(4 \pi t)^{-\frac{d}{2}} e^{-\frac{|x|^{2}}{4 t}}$. The expression $\left(\partial_{t}-\Delta\right)^{-1} \xi$ also acts as an integral operator on $\xi$ via

$$
\left(\partial_{t}-\Delta\right)^{-1} \xi(t, x):=\int_{0}^{t} \int_{[0,1]^{d}} P(t-s, x-y) \xi(d s, d y),
$$

\footnotetext{
${ }^{5}$ As we will primarily work with parabolic equations, these spaces $\mathcal{C}^{\alpha}$ have a built-in parabolic scaling between time and space wherein time regularity is doubled. For instance a $\mathcal{C}^{2}$ function has second continuous spatial derivatives and first continuous time derivative. Extending the situation discussed earlier for $(d=0)$ white noise, we have that space-time white noise $\xi \in \mathcal{C}^{\alpha}$ for $\alpha<-\frac{d+2}{2}$. The case $d=0$ has $\xi \in \mathcal{C}^{-1-}$ which, given the doubling of time regularity, corresponds to the $-\frac{1}{2}-$ regularity discussed above.
} 
which is the space-time convolution of the heat kernel $P(t, x)$ with $\xi$. Just like $\xi$ itself, $\left(\partial_{t}-\Delta\right)^{-1} \xi$ is also a well-defined random distribution.

To get a bit more flavor of "solution theories" of stochastic PDEs, we list some well-known properties for (2.6).

(P1) The solution, in the above sense, is obviously unique, since the difference of two solutions would solve a deterministic heat equation with zero initial condition which must be zero.

(P2) With the aforementioned regularity of $\xi$, by standard parabolic PDE theory, in particular the Schauder estimate which states that the operator $\left(\partial_{t}-\Delta\right)^{-1}$ increases regularity by 2 , one has $u \in \mathcal{C}^{\alpha}$ for any $\alpha<-\frac{d+2}{2}+2=\frac{2-d}{2}$. In particular, $u$ is (almost surely) a random continuous function in $d=1$, and a random distribution in $d \geq 2$. So the limiting polymer parametrized by $x \in[0,1]$ in the above example is a random continuous curve.

(P3) The random distribution $\left(\partial_{t}-\Delta\right)^{-1} \xi$ has Gaussian probability law. This is because $\xi$ is Gaussian and any linear combination of Gaussian random variables is still Gaussian.

(P4) Equation (2.6) has an invariant measure called the Gaussian free field. This is a Gaussian random field on $[0,1]^{d}$ with covariance given by the Green's function of the Laplace $\Delta$. Being invariant means that if the initial data $u_{0}$ is random and distributed as Gaussian free field, then $u(t, \cdot)$ has the same law of Gaussian free field for all $t>0$. On the other hand starting from arbitrary $u_{0}$, the law of $u(t, \cdot)$ will approach that of the Gaussian free field as $t \rightarrow \infty$. We refer to [She07] for a nice review of the Gaussian free field.

(P5) Equation (2.6) is scaling invariant in any dimension $d$, namely,

$$
\widetilde{u}(t, x):=\lambda^{\frac{d-2}{2}} u\left(\lambda^{2} t, \lambda x\right) \quad \text { satisfies } \partial_{t} \widetilde{u}(t, x)=\Delta \widetilde{u}(t, x)+\widetilde{\xi}(t, x),
$$

where $\widetilde{\xi}(t, x):=\lambda^{\frac{d+2}{2}} \xi\left(\lambda^{2} t, \lambda x\right) \stackrel{\text { law }}{=} \xi(t, x)$. The last scaling relation of the white noise can be seen from its covariance (2.8) recalling that the Dirac $\delta$ on $n$-dimensional space has scaling dimension $-n$. Note that the scaling taken in (2.3) and (2.4) was precisely the one that leaves the limit equation invariant.

So far a reader who is new to the area of SPDEs should have acquired the following message: the solution theories of SPDEs share some of the same fundamental challenges as in the study of classical PDEs. These include showing that solutions exist (or can be defined) both locally or globally, are unique within certain regularity classes, and arise as a scaling limits for various approximation schemes. The rest of this article will focus on recent progress on these challenges for nonlinear SPDEs. Before doing so, let us briefly remark on another important challenge present both for PDEs and SPDEs - explicitly representing solutions via formulas.

Linear PDEs always admit explicit solutions. Linear SPDEs, as we saw above, have solutions which are random Gaussian processes with explicit mean and covariance (computable from the equation explicitly). Most nonlinear PDEs do not admit explicit solutions; those that do are generally related to the area of integrable systems. Likewise, most nonlinear SPDEs do not admit explicit descriptions for the probability distribution of their solutions. There are, however, a few special SPDEs (such as the KPZ equation discussed below) which can be explicitly solved in this sense. The study of such SPDEs fall under the area of integrable probability or exactly solvable systems; see for instance [Cor14, BP15] and references therein. We will not pursue this direction further in this article. 

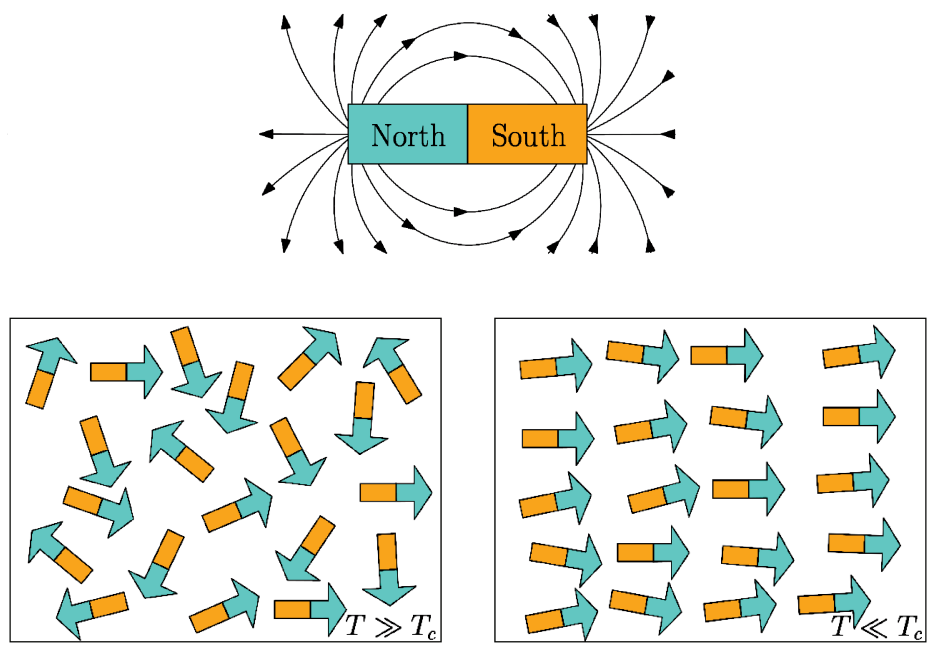

FiguRE 3.1

\section{NONLINEAR SPDES}

Linear systems are often insufficient to effectively model many interesting phenomena. Indeed, as we will now see, nonlinear SPDEs arise in a number of important areas of physics (and many other directions that we will not discuss here). Such nonlinear systems, however, are generally much more challenging to work with. Before coming to that, let us start with a few examples.

Consider a piece of magnet that is being heated up, as in Figure 3.1. As the temperature $T$ increases, the magnetic field produced by the magnet weakens, and at a critical temperature $T_{c}$, known as the Curie temperature 6 the magnetic field disappears. Though various magnet materials have different microscopic structures, a common physical explanation for magnetism is that it comes from the alignment of the magnetic moments of many of the atoms in the material. As a simplified mathematical model one can imagine that a magnet is made up of millions of tiny arrows (or spins) with directions oscillating over time. Below the Curie temperature, i.e., $T<T_{c}$, the spins tend to align in order to minimize an interaction energy (which energetically prefers alignment), which causes a macroscopic magnetization (shown in the bottom-right picture); above the Curie temperature $T>T_{c}$, the spin configurations are much more disordered due to strong thermal fluctuation 7 and as a result the magnetic fields cancel out (shown in the bottom-left picture).

A general mantra in statistical physics holds that "interesting scaling limits arise at critical points". In particular, here we would like to understand what happens to the spin system when the temperature $T$ approaches $T_{c}$ while time and space are tuned accordingly. Near criticality, the spins start to oscillate more and more drastically and the small scale disorder starts to propagate to larger and larger

\footnotetext{
${ }^{6}$ The Curie temperature is named after Pierre Curie who first experimentally demonstrated that certain magnets lost their magnetism entirely at a critical temperature.

${ }^{7}$ In statistical mechanics, thermal fluctuations are random deviations of a system from its low energy state. All thermal fluctuations become larger and more frequent as the temperature increases, and likewise they decrease as temperature approaches absolute zero. Thermal fluctuations are a basic manifestation of the temperature of systems.
} 
scales. The resulting magnetic field fluctuations are believed to be described by the nonlinear SPDE,

$$
\partial_{t} \Phi=\Delta \Phi-\Phi^{3}+\xi
$$

when $\Phi=\Phi(t, x)$ and the spatial dimension of $x$ is 1,2 , or 3 . This is called the dynamical $\Phi^{4}$ equation since the deterministic part arises from the gradient of an energy $\int \frac{1}{2}|\nabla \Phi|^{2}+\frac{1}{4} \Phi^{4} d x$. We will return to this equation later in Section 5.1 and describe how it arises from a particular model of magnets.

As another example, we consider a model for interface growth, where each point of the interface randomly grows up or drops down over time, with a trend to locally smooth out the interface (like the spring force in the polymer example in Section 2). Such systems are ubiquitously found in nature - for instance, the left image in Figure 3.2 shows the end result of an interface grown in the ocean from a volcanic eruption. We are interested in modeling the evolution of such interfaces.
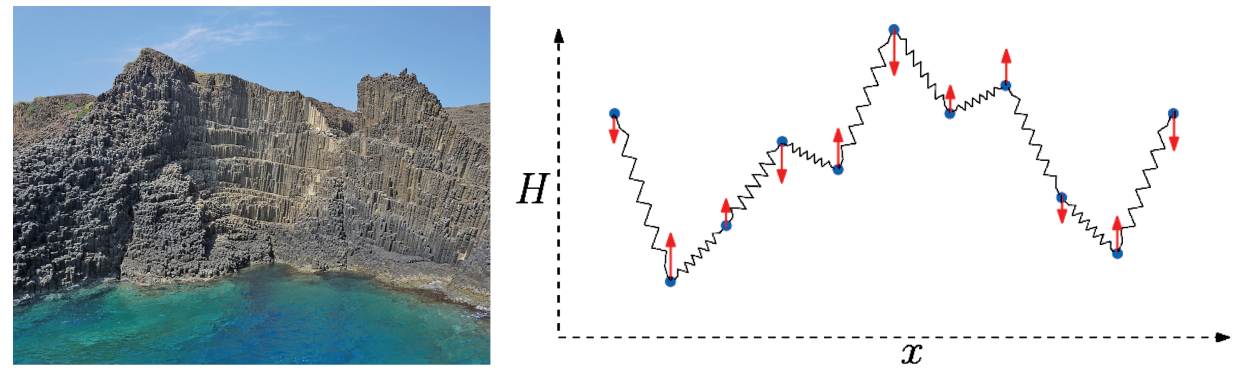

FiguRe 3.2

To drastically simplify the situation, let us assume our interface is a one-dimensional function $H(t, x)$ for $x \in \mathbf{R}$. The simplest scenario is that the upward growth and downward drop of $H$ occurs equally likely, though randomly. In this case, it turns out that the interface behaves similarly to the one-dimensional version of the polymer in Section 2 whose beads are kicked by isotropic random force (see the right image in Figure 3.2). Thus, the large scale fluctuation should be given by the linear SPDE (2.5).

In the asymmetric scenario where the interface is more likely to grow up than to drop down, one expects to see nontrivial fluctuation described by equation (2.5) perturbed by a nonlinearity. In particular, the asymmetry should not be too strong or it will overwhelm the local smoothing (the $\partial_{x}^{2} H$ term) and randomness (the $\xi$ term), and not be too weak or it will not change the limiting equation. This critical tuning is called weakly asymmetric and results (under the same sort of scaling as in the symmetric case) in the following SPDE description for fluctuation of $H(t, x)$ :

$$
\partial_{t} H=\partial_{x}^{2} H+\left(\partial_{x} H\right)^{2}+\xi .
$$

Due to the asymmetry, the interface establishes an overall height shift. So, for the above limit, we must recenter into this moving frame. The KPZ equation was first proposed by Kardar, Parisi, and Zhang in KPZ86; see the nice review KS91b for more background. We will return to this equation later in Section 6 and describe why it arises from various models of interface growth. 
3.1. Some other important nonlinear SPDEs. Besides the $\Phi^{4}$ and (KPZ) equations discussed above, there are a number of physically important equationssome of which we briefly review now. The reader is warned that it is a formidable challenge to define the meaning of a solution to nonlinear SPDEs driven by very singular noises. We postpone this important issue until Section 3.2

- Stochastic Navier-Stokes equation (with spatial dimensions $d=2,3$ of particular physical interest),

$$
\partial_{t} \vec{u}+\vec{u} \cdot \nabla \vec{u}=\Delta \vec{u}-\nabla p+\vec{\zeta}, \quad \operatorname{div} \vec{u}=0,
$$

where $p$ is the pressure and $\vec{\zeta}$ is a $d$-vector valued noise. When the noise $\vec{\zeta}$ is taken to be singular (for instance each component of $\vec{\zeta}$ is an independent space-time white noise), it models motion of fluid with randomness arising from microscopic scales, and in this case we refer to [DPD02, ZZ15] for wellposedness results.

We remark that while this article focuses on singular noises, when modeling large scale random stirring of the fluid, the noise $\vec{\zeta}$ is often assumed to be smooth (it is called colored noise in contrast with white noise), and in fact the most important case is that the equation is driven by only a small number of random Fourier modes. In these situations the long-time behavior is of primary interest, and various dynamical system questions such as ergodicity and mixing are studied. There is a vast literature on this topic, and we only refer to the book [KS12] and the survey articles Mat03, Fla08, Kup10.

- Stochastic heat equation with multiplicative noise in one spatial dimension,

$$
\partial_{t} u=\Delta u+f(u) \xi
$$

where $f$ is some continuous function. The Itô solution theory successful for stochastic ordinary differential equations (ODEs) can extend to this stochastic PDE; see for instance the lecture notes Wal86. The specialization $f(u)=u$, i.e.,

$$
\partial_{t} u=\Delta u+u \xi,
$$

has a significant connection to the KPZ equation: one can formally check that if $H$ solves KPZ, then the Hopf-Cole transform $u:=e^{H}$ solves (3.3). Other choices of $f$ are

$$
f(u)=\alpha \sqrt{u} \quad \text { and } \quad f(u)=\alpha \sqrt{u(1-u)} \quad(\alpha \in \mathbf{R}),
$$

which (along with $f(u)=u$ ) arise in modeling population dynamics and genetics; see for instance [Daw72, Fle75].

- Nonlinear parabolic Anderson model in spatial dimensions $d=2,3$,

$$
\partial_{t} u=\Delta u+f(u) \zeta,
$$

where $f$ is a continuous function and $\zeta$ is a noise which typically is assumed to be spatially independent (i.e., white) but constant in time. This models the motion of mass through random media. The assumption of constant in time noise is consistent with the regime where the mass is assumed to move much faster than the time scale in which the media changes. We refer to GIP15, HL18, and references therein for well-posedness results. 
The parabolic Anderson model, especially the linear case $(f(u)=u)$, is a simple model which exhibits intermittency over long time; for the study of long time behavior, one often considers the spatial-discrete equation with $\zeta$ being independent noises on lattice sites; see, for instance, the reviews CM94] and [K16] for further discussion and references regarding long time behaviors of the parabolic Anderson model.

\section{- Dynamical sine-Gordon equation,}

$$
\partial_{t} u=\Delta u+\sin (\beta u)+\xi, \quad(t, x) \in \mathbf{R}_{+} \times \mathbf{T}^{2} .
$$

This equation describes the natural dynamics of a class of two-dimensional systems that exhibits the Berezinskiu-Kosterlitz-Thouless (BKT) phase transition Ber70, KT73, Jos13, such as two-dimensional Coulomb gas and certain condensed matter materials 8 See [MS77, FS81] for earlier studies of the model in equilibrium. Here $\beta^{2}$ represents the inverse temperature, and $\beta^{2}=8 \pi$ is the BKT critical point. See [HS16, CHS18 for the construction of local solutions of this dynamic.

- Random motion of a curve in an $N$-dimensional manifold $M$ driven by $m$ independent space-time white noises (see [Hai16, BGHZ19, RWZZ18]),

$$
\partial_{t} h^{\alpha}=\partial_{x}^{2} h^{\alpha}+\sum_{\beta, \gamma=1}^{N} \Gamma_{\beta \gamma}^{\alpha}(h) \partial_{x} h^{\beta} \partial_{x} h^{\gamma}+\sum_{i=1}^{m} \sigma_{i}^{\alpha}(h) \xi_{i} \quad \alpha \in\{1,2, \ldots, N\}
$$

where $h$ is a map from an interval to $M, \Gamma_{\beta \gamma}^{\alpha}$ are the Christoffel symbols for the Levi-Civita connection, and $\left\{\sigma_{i}\right\}_{i=1}^{m}$ is a collection of vector fields on the manifold. This is a non-Euclidean generalization of (2.5).

- Stochastic Yang-Mills flow in spatial dimensions $d \leq 4$

$$
\partial_{t} A=-d_{A}^{*} F_{A}+\xi
$$

where the deterministic part (without $\xi$ ) is the Yang-Mills gradient flow introduced in DK90, which is extensively studied in geometry (see the monograph [Fee14]). Here, in the setting of differential geometry, one fixes a Lie group, $A$ is a connection (or a Lie algebra valued 1 -form), $F_{A}$ is the curvature of $A, d_{A}$ is the covariant derivative operator, and $d_{A}^{*}$ is its adjoint. The noise $\xi=\xi_{1} d x_{1}+\cdots+\xi_{d} d x_{d}$ is a 1 -form with each component $\xi_{i}$ being an (independent copy of) Lie algebra valued space-time white noise. See [She18, for some initial progress in $d=2$ in the case that the Lie group is Abelian. Note that (3.7) is not a parabolic equation, and one usually adds an additional term $-d_{A} d_{A}^{*} A$ on the right-hand side to obtain a parabolic equation,

$$
\partial_{t} A=-d_{A}^{*} F_{A}-d_{A} d_{A}^{*} A+\xi
$$

which is gauge equivalent with the original equation (the Donaldson-De Turck trick).

The study of geometric equations with randomness such as (3.6) and (3.7) is of general interest. Equation (3.7) is motivated by the problem of quantization of the Yang-Mills field theory; see also the next item.

\footnotetext{
${ }^{8}$ These include thin disordered super-conducting granular films. The phase transition is from bound vortex-antivortex pairs at low temperatures to unpaired vortices and antivortices at some critical temperature.
} 
- Stochastic quantization. This refers to a large class of singular SPDEs arising from Euclidean quantum field theories defined via Hamiltonians (or actions, energy, etc.). They were introduced by Parisi and Wu in [PW81. Given a Hamiltonian $\mathcal{H}(\Phi)$, which is a functional of $\Phi$, one considers a gradient flow of $\mathcal{H}(\Phi)$ perturbed by space-time white noise $\xi$ :

$$
\partial_{t} \Phi=-\frac{\delta \mathcal{H}(\Phi)}{\delta \Phi}+\xi
$$

Here $\frac{\delta \mathcal{H}(\Phi)}{\delta \Phi}$ is the variational derivative of the functional $\mathcal{H}(\Phi)$; for instance, when $\mathcal{H}(\Phi)=\frac{1}{2} \int(\nabla \Phi)^{2} d x$ is the Dirichlet form, $\frac{\delta \mathcal{H}(\Phi)}{\delta \Phi}=-\Delta \Phi$ and (3.9) boils down to the stochastic heat equation (2.6). Note that $\Phi$ can be also multicomponent fields, with $\xi$ being likewise multicomponent. The aforementioned $\Phi^{4}$ equation, sine-Gordon equation, and stochastic Yang-Mills flow all belong to this class of stochastic quantization equations, each corresponding to a Hamiltonian $\mathcal{H}$.

The significance of these stochastic quantization equations (3.9) is that given a Hamiltonian $\mathcal{H}(\Phi)$, the formal measure

$$
\frac{1}{Z} e^{-\mathcal{H}(\Phi)} D \Phi
$$

is formally an invariant measure 9 for equation (3.9). Here $D \Phi$ is the formal Lebesgue measure and $Z$ is a normalization constant. We emphasize that (3.10) is only a formal measure because, among several other reasons, there is no Lebesgue measure $D \Phi$ on an infinite-dimensional space and it is a priori not clear at all if the measure can be normalized. These measures arise from $\mathrm{Eu}$ clidean quantum field theories. In their path integral formulations, quantities of physical interest are defined by expectations with respect to these measures. The task of constructive quantum field theory is to give precise meaning or constructions to these formal measures; see the book [Jaf00].

Given the very recent progress of SPDEs, a new approach to construct the measure of the form (3.10) is to construct the long-time solution to the stochastic PDE (3.9) and average the distribution of the solution over time. This approach has been shown to be successful for the $\Phi^{4}$ model in $d \leq 3$ in a series of very recent works, which starts with MW17b] on the torus $\mathbf{T}^{3}$ where a priori estimates were obtained to rule out the possibility of finite time blowup. Then GH18b, GH18a] established a priori estimates for solutions on the full space $\mathbf{R}^{3}$ yielding the construction of $\Phi^{4}$ quantum field theory on the whole $\mathbf{R}^{3}$, as well as verification of some key properties that this invariant measure must satisfy as desired by physicists, such as reflection positivity. See also AK17. Similar uniform a priori estimates are obtained by [MW18] using maximum principle.

\section{- Random (nonlinear) Schrödinger equation,}

$$
i \frac{d u}{d t}=\Delta u+\lambda|u|^{2} u+u \xi
$$

\footnotetext{
${ }^{9}$ Being invariant means that if the initial condition of (3.9) is random with probability law given by (3.10), then the solution at any $t>0$ will likewise be distributed according to this same probability law. For readers familiar with stochastic ODEs, one simple example is given by the Ornstein-Uhlenbeck process, $d X_{t}=-\frac{1}{2} X_{t} d t+d B_{t}$, where $B_{t}$ is the Brownian motion, and its invariant measure is the (one-dimensional) Gaussian measure $\frac{1}{\sqrt{2 \pi}} e^{-\frac{X^{2}}{2}} d X$.
} 
where $u$ is complex valued, and $\xi$ is a real valued spatial white noise. The linear case $\lambda=0$ is a model for Anderson localization (a complex version of (3.4); see the recent works [AC15, GKR18]). In the nonlinear case, it describes the evolution of nonlinear dispersive waves in a totally disordered medium, with $\lambda>0$ corresponding to the focusing case and $\lambda<0$ to the defocusing case; see [Con12, $\mathrm{GGF}^{+} 12$ for its physical background and [DW18, DM17, GUZ18] for recent mathematical results.

- (Nonlinear) stochastic wave equation,

$$
\partial_{t}^{2} u-\Delta u+F(u)=\xi
$$

with given initial data $\left.\left(u, \partial_{t} u\right)\right|_{t=0}$. The linear case $(F=0)$ in $d=1$ spatial dimension, as Walsh explained in Wal86], describes "a guitar left outdoors during a sandstorm. The grains of sand hit the strings continually but irregularly." If $\xi(d t, d x)$ is the random measure of the number of grains hitting in $(d t, d x)$ (centered by subtracting the mean), then $\xi$ should be space-time white noise since the numbers hitting over different time intervals or string portions will be essentially independent. The position $u(t, x)$ of the string should satisfy (3.12) with $F=0$.

Equation (3.12) with nonzero $F$ are investigated in earlier works by [AHR96, OR98 in spatial dimensions $d=2,3$, and they proved that with just a function $F$ (satisfying some nice properties) the solution to (3.12) is trivial, namely the same with the solution for $F=0$; the reason for this triviality will be clear in the next subsection.

More recently, GKO18b obtained nontrivial solutions with $F$ given (formally! - see the next subsection) by $F(u)=\lambda u^{k}$ in $d=2$, and $F(u)=u+u^{2}$ in $d=3$ in GKO18a. GUZ18 then studied a stochastic wave equation with $F(u)=u^{3}$ and multiplicative noise ( $u \xi$ on the right-hand side) in $d=2,3$.

Remark 3.1. Note that these nonlinear SPDEs are generally not scaling invariant, unlike the linear stochastic heat equation (2.6), which is scaling invariant in any dimension $d$ (recall property (P5) at the end of Section 2). For instance, for the KPZ equation, $\widetilde{H}(t, x):=\lambda^{\frac{d-2}{2}} H\left(\lambda^{2} t, \lambda x\right)$ will satisfy $\partial_{t} \widetilde{H}=\partial_{x}^{2} \widetilde{H}+\lambda^{\frac{2-d}{2}}\left(\partial_{x} \widetilde{H}\right)^{2}+\widetilde{\xi}$, where $\widetilde{\xi}$ is a new white noise and thus not invariant unless $d=2$. (Indeed, any choice of three scaling components for $t, x, H$ cannot make four terms invariant.) This will turn out to be important for defining solutions to these equations; see Remark 4.2 . Also, as we will see in Sections 5 and [6, it would not be possible to derive these equations (that are not scaling invariant) as limits of scaling certain physical models, unless the physical models have a weak asymmetry, a long interaction range, or a weak intensity of noise, which sets an additional scale.

3.2. Challenge of solution theory. Solution theories for SPDEs have been developed since the 1970s. Earlier progress was recorded by the books written in the 1980s such as [KR82,Wal86], and more recent books such as CR99,PR07,DKM ${ }^{+} 09$, DPZ14,LR15. However, many very important equations, including some of those listed above, remained poorly understood - that is, until very recently.

The difficulty in building solution theories to nonlinear SPDEs is that often these equations are too singular; namely, the solution (if it exists) would have low enough regularity so that certain parts of the equation do not a priori make sense. Indeed, recall that for the linear equation (2.6) in $d$ spatial dimensions, the solution is almost surely an element of $\mathcal{C}^{\alpha}$ for $\alpha<\frac{2-d}{2}$, which is continuous when $d=1$ 
and is a distribution when $d \geq 2$. Since with nonlinear terms the solutions are not expected to be more regular, the " $\Phi^{3}$ " term in the $\Phi^{4}$ equation when $d \geq 2$ is a priori meaningless because distributions in general cannot be multiplied. Similarly, for the KPZ equation, if $d \geq 1, \partial_{x} H$ is distribution valued and thus the term " $\left(\partial_{x} H\right)^{2}$ " does not have a clear meaning. For this type of singular SPDE, it is challenging to even interpret what one means by a solution 10

Starting in the 1980s, the idea of renormalization entered the study of SPDEs JLM85, AR91,DPD03. Recently, this idea has received far-reaching generalization in work by Hairer [Hai14b], Gubinelli, Imkeller, and Perkowski GIP15, and many subsequent works. The idea is to subtract terms with infinite constants from the nonlinearities. Taking the $\Phi^{4}$ equation as an example with spatial dimension $d \leq 3$, one needs to consider the renormalized $\Phi^{4}$ equation,

$$
\partial_{t} \Phi=\Delta \Phi-\left(\Phi^{3}-\infty \Phi\right)+\xi .
$$

This precisely means the following. Since the origin of the problem is the singularity of the driving noise $\xi$, one starts by regularizing $\xi$. For instance, we take a space-time convolution of $\xi$ with a mollifier $\varphi_{\varepsilon}$ that is a smooth function of space and time with support of size $\varepsilon$ so that $\varphi_{\varepsilon} \rightarrow \delta$ (the Dirac delta distribution) as $\varepsilon \rightarrow 0$. Now we consider the $\Phi^{4}$ equation driven by $\xi_{\varepsilon}$,

$$
\partial_{t} \Phi_{\varepsilon}=\Delta \Phi_{\varepsilon}-\Phi_{\varepsilon}^{3}+\xi_{\varepsilon}, \quad \text { where } \xi_{\varepsilon}=\xi * \varphi_{\varepsilon} .
$$

For any $\varepsilon>0$, due to the smoothness of the noise, we can solve the above PDE in the classical sense, and $\xi_{\varepsilon} \in \mathcal{C}^{\infty}$ implies $\Phi_{\varepsilon} \in \mathcal{C}^{\infty}$. As $\varepsilon \rightarrow 0, \xi_{\varepsilon} \rightarrow \xi$, but $\Phi_{\varepsilon}$ do not converge to any nontrivial limit!

The idea is that before the limit, one should insert renormalization terms (also often called counter-terms in the context of quantum field theory 11 )

$$
\partial_{t} \Phi_{\varepsilon}=\Delta \Phi_{\varepsilon}-\left(\Phi_{\varepsilon}^{3}-C_{\varepsilon} \Phi_{\varepsilon}\right)+\xi_{\varepsilon},
$$

where $C_{\varepsilon}$ diverges as $\varepsilon \rightarrow 0$ at a suitable rate. If the sequence of constants $C_{\varepsilon}$ is suitably chosen, the sequence of smooth solutions $\Phi_{\varepsilon}$ of (3.15) will converge to a nontrivial limit as $\varepsilon \rightarrow 0$ :

$$
\Phi=\lim _{\varepsilon \rightarrow 0} \Phi_{\varepsilon} .
$$

This is what we mean by a solution to the (renormalized) $\Phi^{4}$ equation. Note that we do not attempt to make sense of a limiting equation (3.13), but we construct $\Phi$ via by a limit procedure, the limit of solutions to a sequence of regularized and renormalized equations (3.15).

The same renormalization procedure applies to the KPZ equation (in one spatial dimension) and many of the other singular SPDEs listed above.

This discussion prompts several questions:

- Why does $\Phi_{\varepsilon}$ converge, and how does one choose suitable constants $C_{\varepsilon}$ to make this convergence happen? Is the resulting limit unique, or does it depend on the mollification? This is essentially the question of "wellposedness" which will be addressed in Section 4 .

${ }^{10}$ The same issue exists for the dispersive equations. For instance, the solution to the stochastic wave equation (3.12) in spatial dimensions $d \geq 2$ is distributional, and this is exactly the reason that the triviality result by AHR96, OR98 for the nonlinear problem should be expected. Indeed, their proofs are based on a Colombeau distribution machinery.

${ }^{11}$ In fact the corresponding quantum field theory requires a renormalization $\int \frac{1}{2}|\nabla \Phi|^{2}+\frac{1}{4} \Phi^{4}-$ $\frac{C_{\varepsilon}}{2} \Phi^{2} d x$ for dimensions 2 and 3 which is well known in physics. 
- Why are we allowed to "change" the equation by inserting new terms that are not negligible - in fact infinite? We address this renormalization question in Section 5, in which we will see that the SPDEs such as $\Phi^{4}$ arise as scaling limits of physical systems and the renormalization will turn out to have physical meanings in these systems.

- How robust are these singular SPDEs under different approximation schemes? This is a universality question, meaning that one singular SPDE should be able to serve as the continuum large scale description of a class of systems which may have different small scale details. We discuss this in Section 6 .

These questions are of course entangled in many ways. In terms of approximations and convergence, the procedure described as in (3.14) is the simplest way of approximation and approaching a limit, but scaling limits of physical models are essentially also ways of obtaining the limits. In terms of uniqueness, one expects to get the same SPDE limit not only for different choices of mollifications in (3.14) but also via scaling limits of perhaps apparently very different models, which is what universality means. Section 5 below will focus on the meaning of renormalization in physical models, and Section [6 will provide more detailed discussions on deriving an SPDE from these physical models, and of course in all these endeavors one needs to first understand the meaning of a solution as discussed in Section 4.

\section{WELL-POSEDNESS OF SINGULAR SPDES}

We discuss how to choose suitable renormalization constants so that one can obtain a nontrivial limit for solutions to renormalized equations. Our exposition consists of two parts.

- Starting from the 1990s, solutions to renormalized singular SPDEs have been constructed; see for instance [AR91,DPD03. Here we present an elegant argument due to DPD03 which illustrates a simple example of renormalization, plus a standard Picard iteration (fixed point) PDE argument. This argument, despite its simplicity, yields solutions to several singular (but not too singular) equations, such as the $\Phi^{4}$ equation in two spatial dimensions.

- The above argument fails for more singular SPDEs, such as $\Phi^{4}$ in three spatial dimensions and the KPZ equation in one spatial dimension. This motivates us to turn to a more robust approach - the theory of regularity structures introduced in Hai14b. We will also mention some alternative theories or methods, such as paracontrolled distributions GIP15 or renormalization groups Kup16.

To focus our discussion in this section, we will work with the $\Phi^{4}$ equation.

4.1. A PDE argument and renormalization. Consider the $\Phi^{4}$ equation, where the spatial variable takes values in the two-dimensional torus. As explained above, we take a sequence of mollified noises $\xi_{\varepsilon}$ and consider the mollified equation (3.14). 
Write $u_{\varepsilon}:=\left(\partial_{t}-\Delta\right)^{-1} \xi_{\varepsilon}$ for the stationary solution 12 to the mollified linear stochastic heat equation (2.6) $\partial_{t} u_{\varepsilon}=\Delta u_{\varepsilon}+\xi_{\varepsilon}$. The key observation is that the most singular part of $\Phi$ is $u$, so if we write

$$
\Phi_{\varepsilon}=u_{\varepsilon}+v_{\varepsilon},
$$

we can expect the remainder $v_{\varepsilon}$ to converge in a space of better regularity. Subtracting this linear equation from (3.14) gives

$$
\partial_{t} v_{\varepsilon}=\Delta v_{\varepsilon}-\left(v_{\varepsilon}+u_{\varepsilon}\right)^{3}=\Delta v_{\varepsilon}-v_{\varepsilon}^{3}-3 u_{\varepsilon} v_{\varepsilon}^{2}-3 u_{\varepsilon}^{2} v_{\varepsilon}-u_{\varepsilon}^{3} .
$$

This equation looks more promising since the rough driving noise $\xi_{\varepsilon}$ has dropped out. This manipulation has not solved the problem of multiplying distributions, since the limit of $u_{\varepsilon}$ is still a distribution valued in two spatial dimensions (as we discussed earlier - see the fact (P2) in the end of Section 2). However $u_{\varepsilon}$ is a rather concrete object since it is Gaussian distributed ((P3) in the end of Section 2). This makes it possible to study the behavior of $u_{\varepsilon}^{2}$ and $u_{\varepsilon}^{3}$ via probabilistic methods.

As an illustration, consider the expectation

$$
\mathbf{E}\left[u_{\varepsilon}(t, x)^{2}\right]=\int P(t-s, x-y) P(t-r, x-z) \varphi_{\varepsilon}^{(2)}(s-r, y-z) d s d y d r d z,
$$

where $\varphi_{\varepsilon}^{(2)}$ is the convolution of $\varphi_{\varepsilon}$ introduced in (3.14) with itself and $P$ is the heat kernel introduced in (2.10). Due to the singularity of the heat kernel $P$ at the origin, this integral diverges like $O(\log \varepsilon)$ as $\varepsilon \rightarrow 0$ in two spatial dimensions. Denoting $C_{\varepsilon}:=\mathbf{E}\left[u_{\varepsilon}(t, x)^{2}\right]$ (which does not depend on $(t, x)$ by stationarity of $u_{\varepsilon}$ ), this calculation indicates that in (4.2) we should subtract $C_{\varepsilon}$ from $u_{\varepsilon}^{2}$, and subtract $133 C_{\varepsilon} u_{\varepsilon}$ from $u_{\varepsilon}^{3}$.

This amounts to considering the renormalized $\Phi^{4}$ equation

$$
\partial_{t} \Phi_{\varepsilon}=\Delta \Phi_{\varepsilon}-\left(\Phi_{\varepsilon}^{3}-3 C_{\varepsilon} \Phi_{\varepsilon}\right)+\xi_{\varepsilon} .
$$

These renormalized powers of $u_{\varepsilon}$ do converge to nontrivial limits. In fact, thanks to Gaussianity of $u_{\varepsilon}$, given a smooth test function $f$ one can explicitly compute any probabilistic moment of $\left(u_{\varepsilon}^{2}-C_{\varepsilon}\right)(f)$ and prove its convergence. By choosing $f$ from a suitable set of wavelets or Fourier basis, one can apply a version of Kolmogorov's theorem 14 to prove that $u_{\varepsilon}^{2}-C_{\varepsilon}$ and $u_{\varepsilon}^{3}-C_{\varepsilon} u_{\varepsilon}$ converge in $\mathcal{C}^{\alpha}$ for any $\alpha<0$. We denote these limits $: u^{2}$ : and $: u^{3}$ : . They are elements of $\mathcal{C}^{\alpha}$ for any $\alpha<0$.

To summarize, we have found that the renormalization constants can be found through explicit moment calculations/expectations.

Passing (4.2) to the limit, we get

$$
\partial_{t} v=\Delta v-\left(v^{3}+3 u v^{2}+3: u^{2}: v+: u^{3}:\right) .
$$

We can prove local well-posedness of this equation as a classical PDE, by a standard fixed point argument. For this, we use a classical result in harmonic analysis under

\footnotetext{
${ }^{12}$ This corresponds to dropping the term involving initial data in 2.9 and integrating time in (2.10) from $-\infty$ instead of 0 . Stationarity means that the distribution of $u_{\varepsilon}$ does not depend on $t$. This assumption will be convenient when performing moment calculations, such as (4.3). Namely, the moments will not depend on space-time points.

${ }^{13}$ The factor 3 arises from three ways of choosing two powers of $u_{\varepsilon}$ from the cubic term $u_{\varepsilon}^{3}$. A Wick theorem allows one to compute expectation of a product of arbitrarily many Gaussian variables, and in two dimensions the Wick renormalized power $: u^{n}:=\lim _{\varepsilon \rightarrow 0} C_{\varepsilon}^{\frac{n}{2}} H_{n}\left(u_{\varepsilon} / \sqrt{C_{\varepsilon}}\right)$.

${ }^{14}$ This is a version of Kolmogorov's theorem (formulated differently as the classical Kolmogorov theorem) which is adapted to prove convergences in the $\mathcal{C}^{\alpha}$ spaces in the present context.
} 
the name Young's theorem 15 which states that if $f \in \mathcal{C}^{\alpha}, g \in \mathcal{C}^{\beta}$, and $\alpha+\beta>0$, then $f \cdot g \in \mathcal{C}^{\min (\alpha, \beta)}$. Thus if we assume that $v \in \mathcal{C}^{\beta}$ for, say, $\beta=1$, then the worst term in the parenthesis in (4.5) has regularity $\alpha$. By the classical Schauder estimate, which states that the heat kernel improves regularity by 2 , the fixed point map

$$
v \mapsto\left(\partial_{t}-\Delta\right)^{-1}\left(v^{3}+3 u v^{2}+3: u^{2}: v+: u^{3}:\right)
$$

is well defined. Namely, it maps a generic element $v \in \mathcal{C}^{\beta}$ to a new element which is again in $\mathcal{C}^{\beta}$ for $\beta=1$. This is since for $\alpha<0$ sufficiently close to 0 one has $\mathcal{C}^{\alpha+2} \subset \mathcal{C}^{\beta}$. With a bit of extra effort, one can show that over a short time interval the fixed point map is contractive and thus has a fixed point in $\mathcal{C}^{\beta}$, and this fixed point $v$ is the solution. (The sharp result is $\mathcal{C}^{\beta}$ for any $\beta<2$.) To conclude, one has $\Phi=u+v$, which is the local solution to the renormalized $\Phi^{4}$ equation in two spatial dimensions.

The above argument was first used by Da Prato and Debussche in DPD03, and it applies to other equations, for instance the stochastic Navier-Stokes equation (3.1) with space-time white noise on a two-dimensional torus DPD02. Let us mention another, somewhat surprising application, that is the dynamical sineGordon equation (3.5) in two spatial dimensions in the regime $\beta^{2} \in(0,4 \pi)$. The renormalized equation reads

$$
\partial_{t} u_{\varepsilon}=\frac{1}{2} \Delta u_{\varepsilon}+C_{\varepsilon} \sin \left(\beta u_{\varepsilon}\right)+\xi_{\varepsilon}, \quad \beta \in \mathbf{R},
$$

where $C_{\varepsilon}$ is a renormalization constant which diverges like $O\left(\varepsilon^{-\frac{\beta^{2}}{4 \pi}}\right)$. By writing $u_{\varepsilon}=\phi_{\varepsilon}+v_{\varepsilon}$ with $\phi_{\varepsilon}:=\left(\partial_{t}-\Delta\right)^{-1} \xi_{\varepsilon}$, one finds that

$$
\partial_{t} v_{\varepsilon}=\frac{1}{2} \Delta v_{\varepsilon}+C_{\varepsilon} \sin \left(\beta \phi_{\varepsilon}\right) \cos \left(\beta v_{\varepsilon}\right)+C_{\varepsilon} \cos \left(\beta \phi_{\varepsilon}\right) \sin \left(\beta v_{\varepsilon}\right) \text {. }
$$

[HS16] proved that $C_{\varepsilon} e^{ \pm i \beta \phi_{\varepsilon}}=C_{\varepsilon}\left(\cos \left(\beta \phi_{\varepsilon}\right) \pm i \sin \left(\beta \phi_{\varepsilon}\right)\right)$ converges to a nontrivial limit in $\mathcal{C}^{-\frac{\beta^{2}}{4 \pi}}$, so $\beta^{2} \in(0,4 \pi)$ is precisely $\sqrt{16}$ the regime where the above classical PDE argument applies. Note that the constant $C_{\varepsilon}$ can be again found by calculating the expectation of $e^{ \pm i \beta \phi_{\varepsilon}}$, i.e., the characteristic function of the Gaussian random variable $\phi_{\varepsilon}$.

The same idea (but with a slightly different transformation than (4.1)) applies to the linear parabolic Anderson model in [HL15]:

$$
\partial_{t} u_{\varepsilon}=\Delta u_{\varepsilon}+u_{\varepsilon}\left(\zeta_{\varepsilon}-C_{\varepsilon}\right), \quad(t, x) \in \mathbf{R}_{+} \times \mathbf{T}^{2},
$$

where $\zeta_{\varepsilon}$ is regularized spatial white noise on $\mathbf{T}^{2}$. With a transformation $v_{\varepsilon}=u_{\varepsilon} e^{Y_{\varepsilon}}$, where $\Delta Y_{\varepsilon}=\zeta_{\varepsilon}$, one can simply check

$$
\partial_{t} v_{\varepsilon}=\Delta v_{\varepsilon}+v_{\varepsilon}\left(\left|\nabla Y_{\varepsilon}\right|^{2}-C_{\varepsilon}\right)-2 \nabla Y_{\varepsilon} \cdot \nabla v_{\varepsilon} .
$$

Again, $Y_{\varepsilon}$ is a Gaussian process, and with $C_{\varepsilon}:=\mathbf{E}\left|Y_{\varepsilon}\right|^{2}=-\frac{1}{2 \pi} \log \varepsilon+O(1)$, $\left|\nabla Y_{\varepsilon}\right|^{2}-C_{\varepsilon}$ converges to a nontrivial limit, and the equation for $v$ is shown to be locally well-posed by standard PDE methods as above. (In fact HL15] constructed a global solution on $\mathbf{R}_{+} \times \mathbf{R}^{2}$, making use of the linearity of the equation.) [DW18]

\footnotetext{
${ }^{15}$ Note that the original form of Young's theorem is only for one-dimensional functions of finite $p$-variations, and the version of Young's theorem we are referring to here can be found in Hai14b, Proposition 4.14] and [BCD11 Theorems 2.47 and 2.52], for instance.

${ }^{16}$ Recall that, for the fixed point argument to work, we must have that the regularity plus 2 is at least 1.
} 
studies the stochastic Schrödinger equation (3.11) on $\mathbf{T}^{2}$, in which a transformation similar to that in HL15] can be applied.

This type of strategy has also been applied to stochastic hyperbolic equations. Consider the stochastic nonlinear wave equations,

$$
\partial_{t}^{2} u-\Delta u \pm u^{k}=\xi, \quad(x, t) \in \mathbf{R}_{+} \times \mathbf{T}^{2},
$$

with given initial data $\left.\left(u, \partial_{t} u\right)\right|_{t=0}$, where $\xi$ is the space-time white noise on $\mathbf{R}_{+} \times \mathbf{T}^{2}$, and $k \geq 2$. GKO18b] adopts the above Da Prato-Debussche trick to write $u$ as a linear part plus a remainder. Such an idea previously appears in the context of deterministic dispersive PDEs with random initial data in earlier work of McKean McK95] and Bourgain Bou96. The proof in GKO18b is based on a fixed point argument for the remainder equation (as above), but with the Schauder estimates replaced by Strichartz estimates for the wave equations. The key point is to use function spaces where the wave equation allows for a gain in regularity. This gain is sufficient to prove that the remainder has better regularity than the linear solution and gives a well-defined nonlinearity for which suitable local-in-time estimates can be established.

4.2. Regularity structures and paracontrolled distributions. The above argument fails for the $\Phi^{4}$ equation in three spatial dimensions. As dimension increases, the space-time white noise (and thus the solution) becomes more singular. To see how this problem rears its head, consider the term $: u^{2}: v$ in (4.5). One can show 17 that in three spatial dimensions, as a space-time distribution, : $u^{2}: \in \mathcal{C}^{\alpha}$ for any $\alpha<-1$. Thus, to multiply : $u^{2}$ : with $v$, we would have to formulate the fixed point map (4.6) for $v \in \mathcal{C}^{\beta}$ with $\beta>1$. The product $: u^{2}: v$ would then lie in $\mathcal{C}^{\alpha}$ for any $\alpha<-1$. Unfortunately, $\left(\partial_{t}-\Delta\right)^{-1}$ only provides two more degrees of regularities, and thus the fixed point map (4.6) will not bring an element in $\mathcal{C}^{\beta}$ back to the same space.

A natural idea is to go one step further in the expansion (4.1). In view of the equation (4.2), we define a second order perturbative term, $w_{\varepsilon}:=\left(\partial_{t}-\Delta\right)^{-1}: u_{\varepsilon}^{3}$ : , and rewrite the expansion as

$$
\Phi_{\varepsilon}=u_{\varepsilon}-w_{\varepsilon}+R_{\varepsilon} .
$$

It turns out that one can prove that $w_{\varepsilon}$ converges in $\mathcal{C}^{\alpha}$ for $\alpha<1$ to a limit $w$. It remains to see whether $R_{\varepsilon}$ converges to a limit $R$ with even better regularity. Using (4.4), it is straightforward to derive an equation for $R_{\varepsilon}$ :

$$
R_{\varepsilon}=\left(\partial_{t}-\Delta\right)^{-1}\left(-3\left(u_{\varepsilon}^{2}-C_{\varepsilon}\right) R_{\varepsilon}-3\left(u_{\varepsilon}^{2}-C_{\varepsilon}\right) w_{\varepsilon}+\cdots\right) .
$$

There should be eight terms in the parenthesis, but we have only written down the two terms that are important for our discussion; the other terms (in "...") can be treated by the standard PDE argument as in the two-dimensional case above. It turns out that even after this higher order expansion (4.7), the above PDE fixed point argument still does not work because of the two terms written on the righthand side of (4.8).

For the second term, $\left(u_{\varepsilon}^{2}-C_{\varepsilon}\right) w_{\varepsilon}$, we have $u_{\varepsilon}^{2}-C_{\varepsilon} \rightarrow: u^{2}: \in \mathcal{C}^{-1-}$ and $w_{\varepsilon} \rightarrow$ $w \in \mathcal{C}^{\frac{1}{2}-}$. This is below the borderline of applicability of Young's theorem. It is not hard to overcome this difficulty. In fact, in three spatial dimensions, the

\footnotetext{
${ }^{17}$ In three spatial dimensions $\xi \in \mathcal{C}^{-\frac{5}{2}-}$, therefore $u \in \mathcal{C}^{-\frac{1}{2}-}$. From this one can show that $: u^{2}: \in \mathcal{C}^{-1-}$ (the rigorous proof of this fact is done by moment analysis).
} 
term $\left(u_{\varepsilon}^{2}-C_{\varepsilon}\right) w_{\varepsilon}$ requires further renormalization to converge to a nontrivial limit. Since this term is nothing but a convolution of several heat kernels and Gaussian noises, one can again carry out a moment analysis to find a suitable renormalization constant $\bar{C}_{\varepsilon}$, which turns out to diverge logarithmically such that

$$
\left(u_{\varepsilon}^{2}-C_{\varepsilon}\right) w_{\varepsilon}-\bar{C}_{\varepsilon} u_{\varepsilon} \quad \text { converges in } \mathcal{C}^{-\frac{1}{2}-} .
$$

This amounts to renormalizing the $\Phi^{4}$ equation in three spatial dimensions as

$$
\partial_{t} \Phi_{\varepsilon}=\Delta \Phi_{\varepsilon}-\left(\Phi_{\varepsilon}^{3}-\left(3 C_{\varepsilon}+\bar{C}_{\varepsilon}\right) \Phi_{\varepsilon}\right)+\xi_{\varepsilon},
$$

where $C_{\varepsilon} \sim \varepsilon^{-1}$ and $\bar{C}_{\varepsilon} \sim|\log \varepsilon|$. See also Remark 4.1.

The first term, $-3\left(u_{\varepsilon}^{2}-C_{\varepsilon}\right) R_{\varepsilon}$, is of the same nature as $: u^{2}: v$ in (4.6), so we suffer exactly the same vicious circle of difficulty as in the discussion for $: u^{2}: v$; namely, the fixed point argument does not close. In fact, higher order expansions beyond (4.7) will always end up with such a term so the same problem will remain. This is the real obstacle. The idea of regularity structures (which overcomes this obstacle) is that the solutions to the two equations,

$$
R=\left(\partial_{t}-\Delta\right)^{-1}\left(-3: u^{2}: R\right), \quad \widetilde{R}=\left(\partial_{t}-\Delta\right)^{-1}\left(-3: u^{2}:\right),
$$

should have the same small scale behavior, because $: u^{2}$ : is more singular than $R$ and it is the factor $: u^{2}$ : that dominates the small scale roughness. (Here we have ignored all the other terms in (4.8), which have better regularities than that of $: u^{2}: R$, in order to focus on the main issue of the problem.) This a priori knowledge that $R$ should locally look like $\widetilde{R}$ can be formulated as that when the space-time points $z$ and $z_{0}$ are close, one should expect that 18

$$
R(z)-R\left(z_{0}\right)=R\left(z_{0}\right)\left(\widetilde{R}(z)-\widetilde{R}\left(z_{0}\right)\right)+O\left(\left|z-z_{0}\right|\right) .
$$

Namely, the local increment of $R$ is approximately the same as the local increment of $\widetilde{R}$ - up to multiplying a factor $R\left(z_{0}\right)$ which depends on the base point $z_{0}$; the reason that this multiplicative factor should be $R\left(z_{0}\right)$ is clear from the structure of equation (4.11).

Since $\widetilde{R}$ is again a concrete object, which is simply convolutions of heat kernels with powers of Gaussians, it is easy to prove $\widetilde{R} \in \mathcal{C}^{1-}$ by analyzing its moments as before. Thus if $R$ satisfies (4.12), that is, locally looks like $\widetilde{R}$, one has $R \in \mathcal{C}^{1-}$ as well. The converse is not true; the set of $R$ satisfying (4.12) is a strictly smaller set than $\mathcal{C}^{1-}$. The key is to formulate a fixed point problem in the space of all functions $R$ that have prescribed local expansion (4.12) (rather than in standard function spaces such as $\left.\mathcal{C}^{\alpha}\right)$. The aforementioned vicious term $: u^{2}: R$, which could not be defined for arbitrary $R \in \mathcal{C}^{1-}$, can now be defined if $R$ locally looks like $\widetilde{R}$, because $: u^{2}: \widetilde{R}$ is again simply a concrete combination of Gaussian processes and heat kernels! It turns out that the fixed point argument closes in the space of functions having such prescribed local expansions, and the fixed point $R$ together with (4.7) yields the solution to the $\Phi^{4}$ equation in three dimensions.

The above idea of solving stochastic equations in a space of functions or distributions that have prescribed local approximations by certain canonical objects to

\footnotetext{
${ }^{18}$ Equation (4.12) is reminiscent of a Taylor expansion $F(z)=F\left(z_{0}\right)+F^{\prime}\left(z_{0}\right)\left(z-z_{0}\right)+\cdots$, where one approximates a differentiable function by Taylor polynomials. Here we approximate $R$ by $\widetilde{R}$ which is also an object that is simply a convolution of heat kernels with white noises. Taylor polynomials are special examples of regularity structures, and the theory of regularity structures is a generalization of Taylor expansion.
} 
some extent had its precursor in the simpler setting of stochastic ordinary differential equations, which is called rough path theory (see Lyo98 or the book [FH14]) in particular a formulation by Gub04]. Constructing the solution to the $\Phi^{4}$ equation on a three-dimensional torus was the first example of the theory built in Hai14b. The review articles [Hai15a, Hai15b] have more detailed pedagogical explanations on the theory and the application to this equation.

Remark 4.1. We have found the renormalization of $u_{\varepsilon}^{2}, u_{\varepsilon}^{3}$ and $u_{\varepsilon}^{2} w_{\varepsilon}$ and proved convergence of the renormalized objects by moment analysis. Analyzing moments of these random objects are the only probabilistic component of Hai14b. As the equation in question becomes more singular, the number of such random objects to be studied increases, and it is tedious or even impossible to analyze each of them by hand. [CH16] develops a blackbox that provides systematic and automatic treatment for renormalization and moment analysis for these perturbative objects arising from general singular SPDEs. Moreover, there are also algebraic aspects for the renormalization procedure (so-called renormalization groups), which has been systematically treated in [BHZ16]. Finally, there is a question regarding what the renormalized equation (e.g., (4.10) ) will look like after renormalizing these random objects, and this is answered in [BCCH17].

Hairer's theory has been applied to provide solutions to other very singular SPDEs, for instance, a generalized parabolic Anderson model (a generalization of (3.4),

$$
\partial_{t} u=\Delta u+\sum_{i j} f_{i j}(u) \partial_{i} u \partial_{j} u+g(u) \xi
$$

where $f$ and $g$ are sufficiently regular functions. The well-posedness of the KPZ equation in one spatial dimension was solved in Hai13 using the theory of controlled rough paths Gub04, which can now be viewed as a special case of regularity structures; see the book [FH14] for rough paths, regularity structures, and applications to KPZ.

Other applications include (but are not limited to) the stochastic Navier-Stokes equation (3.1) with white noise on the three-dimensional torus [ZZ15, the stochastic heat equation with multiplicative noise (3.2) [HP15, HL18], the dynamical sine-Gordon equation (3.5) on two-dimensional torus for arbitrary $\beta^{2}<8 \pi$ [HS16. CHS18, the stochastic quantization of Abelian gauge theory/stochastic gauged Ginzburg-Landau equation by [She18, and the random motion of string in manifold (3.6) BGHZ19.

Besides Hairer's theory Hai14b, some alternative methods have also been introduced. The paracontrolled distribution method of Gubinelli, Imkeller, and Perkowski GIP15] is based on a similar idea of controlling the local behavior of solutions, but it is implemented in a different way by using Littlewood-Paley theory and paraproducts BCD11. See GP18b for a review on paracontrolled distribution. The paracontrolled distribution method has been also successfully applied to, for instance, the KPZ equation GP17b, Hos18 in $d=1$ (and more recently the construction of a solution on the entire real line instead of a circle [PR18], a multi-component coupled KPZ equation [FH17, and the $\Phi^{4}$ equation [CC18b] in $d=3$ (and more interestingly, its global solution by MW17b]).

The paracontrolled distribution method has not only allowed us to prove wellposedness results for stochastic PDEs, but it also resulted in the construction of other singular objects that could not be made sense of before. For instance, AC15] 
constructed the Anderson Hamiltonian (i.e., Schrödinger operator) on the twodimensional torus, formally defined as $\mathscr{H}=-\Delta+\xi$, where $\xi$ is a singular potential such as white noise. As another example, CC18a proved existence and uniqueness of solution for stochastic ordinary differential equations $d X_{t}=V\left(t, X_{t}\right) d t+d B_{t}$ with distributional valued drift $V$, where $B$ is a $d$-dimensional Brownian motion, and this is achieved via the study of the generator of the above stochastic ordinary differential equations given by $\partial_{t}+\frac{1}{2} \Delta+V \cdot \nabla$. CC18a also managed to make sense of a singular polymer measure on the space of continuous functions formally given by $\mathbb{Q}_{T}(d \omega)=Z_{0}^{-1} \exp \left(\int_{0}^{T} \xi\left(\omega_{s}\right) d s\right) \mathbb{W}_{T}(d \omega)$, where $\mathbb{W}$ is the Wiener measure (i.e., the Gaussian measure for Brownian motions) on $C\left([0, T], \mathbf{R}^{d}\right)$ for $d=2,3, \xi$ is a spatial white noise on the $d$-dimensional torus independent of $\mathbb{W}$, and $Z_{0}$ is an (infinite) renormalization constant.

We will discuss another application of the paracontrolled distribution method on the scaling limit problem with a bit more detail in Section 5.2.

In the line of this paracontrolled distribution approach, BB16 provided a semigroup approach, which has been applied to the generalized parabolic Anderson model (3.4) on a potentially unbounded two-dimensional Riemannian manifold.

Another method based on renormalization group flow was introduced by Kupiainen Kup16, which for instance has been applied to prove local well-posedness for a generalized KPZ equation KM17] introduced by H. Spohn Spo14 in the context of stochastic hydrodynamics.

With all these alternative methods, the theory of regularity structures is by far the most systematic and general approach; for instance it has developed the blackbox theorems as mentioned in Remark 4.1 which makes the implementation of this theory very automatic, and it can deal with equations which are extremely singular (that is, very close or even arbitrarily close to criticality, see Remark 4.2) such as the random string in a manifold (3.6) or the dynamical sine-Gordon equation (3.5) for arbitrary $\beta^{2}<8 \pi$.

Remark 4.2 (Subcriticality of stochastic PDE). The methods developed in Hai14b, [BCD11], and Kup16 are all for subcritical semilinear stochastic PDEs. For stochastic PDEs with white noise, the equation being subcritical means that the nonlinear term has better regularity than the linear terms; namely, small scale roughness is dominated by the linear solution. For instance, for the $\Phi^{4}$ equation in three spatial dimensions, the term $\Phi^{3}$ has regularity $-\frac{3}{2}-$ while $\Delta \Phi$ and $\xi$ have regularities $-\frac{5}{2}-$. Subcriticality often depends on spatial dimensions: (KPZ), (3.2), and (3.6) are subcritical in $d<2$, while $\left(\Phi^{4}\right.$ ), the parabolic Anderson model (3.4), the Navier-Stokes equation (3.1) with space-time white noise, and the stochastic Yang-Mills heat flow (3.8) are subcritical in $d<4$. The dynamical sine-Gordon equation (3.5) however is subcritical for $\beta^{2}<8 \pi$.

The stochastic PDEs being discussed here in supercritical regimes (i.e., above the aforementioned criticalities) are not expected to have nontrivial meanings of solutions. We only expect to get Gaussian limit, although the Gaussian variances may be nontrivial; the reader is referred to [MU18, Theorem 1.1] for a flavor of such a result for the KPZ equation in $d \geq 3$.

Critical dimensions are much more subtle. We refer to CD18, CSZ17b, CSZ18, Gu18 for the very new progress on the KPZ equation in $d=2$. 
Remark 4.3. We remark that although we have focused on semilinear equations in our expositions, the methods developed in Hai14b, BCD11 have also extended to quasilinear equations; see OW18, FG19, BDH19, GH17b].

4.3. A brief discussion on weak solutions. The solutions to SPDEs that we have discussed so far are called strong solutions, as opposed to the weak solutions that we will now briefly discuss 19 Let us immediately point out that the weak solutions in the stochastic context have nothing to do with the weak solutions in deterministic PDE theory; one sometimes adopts the terminology "probabilistically weak solutions".

For a strong solution, one starts with a probability space on which the noise is defined and then builds a mapping from that probability space and the initial data space to a space of functions (or distributions) that satisfies the prescribed equation 20 with probability 1 (i.e., for almost every point in the probability space). Though subtle, it is important to understand that a strong solution to an SPDE need not be function valued (as we saw, in some instances it is distribution valued, living in some spaces of negative regularity).

For a standard PDE, a weak solution requires that the equation holds when tested against a suitable class of functions. For SPDEs, the analogue of this involves treating solutions statistically as probability measures on the solution space, rather than as random variables supported on the probability space on which the noise is defined. Roughly, a weak solution means that we can define some noise (with the right distribution and measurability assumptions satisfied) so that the canonical proces:21 on the solution space, along with the noise satisfying the desired equation 22 As we will see, martingale problems provide a very convenient way to demonstrate that a weak solution solves an SPDE (instead of demonstrating the existence of a suitable noise as above).

Let us illustrate these ideas in the simplest setting of stochastic differential equations (SDEs). Let $\xi(t)$ denote temporal white noise. Like its space-time counterpart, this can be defined in various ways (e.g., as a series with random coefficients). Consider the SDE $d X_{t}=\xi(t) d t$, which in integrated form reads $X_{t}=X_{0}+\int_{0}^{t} \xi(d s)$ (let us assume that $X_{0}=0$ for simplicity). Once integration with respect to white noise is understood, this defines a solution map (and hence a strong solution) from

\footnotetext{
${ }^{19}$ Not all equations that admit weak solutions admit strong solutions. A famous stochastic differential equation example is called Tanaka's equation; see KS91a, Example 3.5].

${ }^{20} \mathrm{As}$ we saw, making sense of what it means to satisfy the equation often takes significant work and involves regularizations and renormalizations. There are also some measurability assumptions which should be imposed on strong solutions so future noise cannot effect the evolution before its time.

${ }^{21}$ The canonical process is the random variable whose probability space is defined as the solution space equipped with the probability measure of the proposed solution.

${ }^{22}$ Here is a, hopefully, more intuitive explanation for this different notion of strong versus weak. Imagine that human life were governed by an SPDE. Then, a strong solution would tell us how each individual's life would unfold, given the knowledge of all of the randomness which befalls them, in addition to the world around them. A weak solution is statistical - it tells us that people with certain characteristics have certain probabilities of having their life unfold in various ways. Given such a prescription of probabilities, how can be verify that this is, indeed, a weak solution to the "SPDE of life"? Well, we need to demonstrate that there exists randomness which would, in fact, result in the aforementioned probabilities. Then, we would need to verify that the randomness is distributed in the way that the SPDE of life claims (e.g., space-time white noise). While this is all a bit tongue-in-cheek, we hope it helps explain the difference.
} 
$\left(\xi, X_{0}\right)$ to the full trajectory of $X_{t}$ for $t \geq 0$. One checks that $t \mapsto X_{t}$ is continuous and that its marginal distributions are Gaussian with covariance of $X_{s}$ and $X_{t}$, given by the minimum of $s$ and $t$. This, in fact, implies that the distribution of the function $t \rightarrow X_{t}$ is a Wiener measure - that is, the distribution of Brownian motion. If instead of $X_{t}$ we had another Brownian motion $\widetilde{X}_{t}$ (for instance, we could have $\tilde{X}_{t}=-X_{t}$ or just an independent Brownian motion), then $\widetilde{X}_{t}$ would be a weak solution, but not a strong solution. This is because $X_{t}$ and $\widetilde{X}_{t}$ have the same distribution, even if they are not "driven" by the same noise.

The martingale problem provides an alternative characterization to the Gaussian description above for Brownian motion. The Lévy characterization theorem says that $X_{t}$ is distributed as a Brownian motion if it is almost surely continuous and both $X_{t}$ and $X_{t}^{2}-t$ are martingales 23 A measure on $X_{t}$ that satisfies this is said to satisfy the martingale problem characterizing Brownian motion.

What does it mean that $X_{t}$ (or $X_{t}^{2}-t$ ) is a martingale? Roughly speaking, this means that given the history of $X_{t}$ up to time $t$, the expected value of its future location is exactly $X_{t}$. This is like a fair gambling system in which your future expected profit is always zero. Martingales are essentially a particular class of centered noise.

Martingale problems exist for general classes of SDEs and are often very useful for proving convergence results. For instance, to show that a discrete time Markov chain converges to an SDE (e.g., a random walk converges to Brownian motion), one can demonstrate that the discrete chain satisfies a discrete version of the SDE's martingale problem. Then, provided one can demonstrate compactness of the measures (on the evolution of the Markov chain), all limit points must satisfy the limiting SDE's martingale problem. This generally proves uniqueness of the limit points and, hence, convergence.

Weak solutions to linear SPDEs can also be characterized in terms of martingale problems. Let us describe how this works for the multiplicative noise stochastic heat equation (3.3), recalled here 24

$$
\partial_{t} u(t, x)-\Delta u(t, x)=u(t, x) \xi(t, x) \quad(t, x) \in \mathbf{R}_{+} \times \mathbf{R} .
$$

Let us write $u_{t}(x)=u(t, x)$ and think of $u$ as a measure on $C\left(\mathbf{R}_{+}, C(\mathbf{R})\right.$ ) (continuous maps from $t \in \mathbf{R}_{+}$to continuous spatial functions). For any test function $\varphi \in C^{\infty}(\mathbf{R})$, write $u_{t}(\phi)=\int u_{t}(x) \phi(x) d x$. With this notation, define the processes $(4.14)$

$$
N_{t}(\varphi):=u_{t}(\varphi)-u_{0}(\varphi)-\int_{0}^{t} u_{s}(\Delta \varphi) d s \quad \text { and } \quad Q_{t}(\varphi):=N_{t}(\varphi)^{2}-\int_{0}^{t}\left(u_{s}^{2}, \varphi^{2}\right) d s .
$$

We say that $u$ satisfies the martingale problem for the multiplicative noise stochastic heat equation if both $N_{t}$ and $Q_{t}$ are (local) martingales for all test functions $\phi$. Any $u$ that satisfies this is a weak solution; see for instance [BG97, Definition 4.10]. Just as martingale problems are useful in proving convergence of Markov chains to SDEs, so too can they be used in SPDE convergence proofs; see Sections 6.2 and 6.3 for some examples where this type of martingale problem has been used for such a purpose.

\footnotetext{
${ }^{23}$ In fact, local martingales.

${ }^{24}$ This equation also admits a strong solution which can be written as a chaos series of multiple stochastic integrals against space-time white noise $\xi$.
} 
It is generally hard to formulate a martingale problem characterization for weak solutions to singular nonlinear SPDEs. For the KPZ equation (in one spatial dimension) $H$, one can use the Hopf-Cole transform and define $H$ as a Hopf-Cole solution to the KPZ equation if $u=e^{H}$ is a solution to the multiplicative noise stochastic heat equation. This notion of solution agrees with those discussed earlier in this text. However, such linearizing transformations are uncommon, and this should be thought of as a rather useful trick, not a general theory.

Remarkably, for the stochastic Burgers equation (which is formally the equation satisfied by the spatial derivative of the KPZ equation $\partial_{x} H$ )

$$
\partial_{t} u=\frac{1}{2} \partial_{x}^{2} u-\frac{1}{2} \partial_{x}\left(u^{2}\right)+\partial_{x} \xi,
$$

GJ14 found a way to formulate a martingale problem characterization and GP17a (with a slightly improved formulation) matches the solution to this martingale problem to the Hopf-Cole solution (see (3.3)) whereby showing uniqueness of the solution to this martingale problem; they call it an energy solution. There were some limitations of this notion of solution, namely it only works for particular types of initial data; very recently, however, GP18c has generalized the notion of energy solution to system configurations with finite entropy with respect to stationarity; and Yan18 has extended this method to include more general initial data such as flat initial data. It has proved to be quite useful in demonstrating convergence results, as we explain later in Section 6.4. Finally, let us mention that very recently GP18a developed a martingale approach for a class of singular stochastic PDEs of Burgers type, including fractional and multicomponent Burgers equations.

Let us end this discussion by mentioning (without any explanation) another powerful approach to defining weak solutions of SPDEs-Dirichlet forms. For example, for the $\Phi^{4}$ equation in two spatial dimensions, before Da Prato and Debussche constructed their strong solution in [DPD03, the paper [AR91] constructed a weak solution via Dirichlet forms (which involves significant functional analysis). For comprehensive discussion on this topic we refer to the book [FOT11 and the references therein.

\section{RenORMALIZATION IN PHYSiCAL MODELS}

Let us take stock of what we have learned so far. In Section 2 we observed that (at least in the linear case) SPDEs arise as scaling limits for microscopic models of physical systems. In Section 3 we introduced a number of nonlinear SPDEs and claimed that they model various interesting physical systems. However, before trying to justify that claim, we had to confront the challenge of well-posedness. Namely, how to make sense of what a "solution" means to these equations. Section 4 surveyed the main techniques for doing this.

In defining a solution, we mollified (or smoothed) the noise and defined the solution through a limit transition. From that perspective, it is reasonable to hope that the same methods can be applied to other types of regularizations of the noise, or equation-for instance to show that discrete systems converge to the SPDEs. We will address this further in Section 6 .

In Section 4 we found that besides regularizing the noise, we also had to introduce certain renormalizations to our equations for them to admit limits. At first glance this tweaking of the equation seems a bit crooked. In this section we will explain how these renormalizations have concrete physical meaning, thus justifying our 
definitions. For instance, a diverging renormalization constant may relate to a tuning for a microscopic system of the overall scale, reference frame, temperature, or other physically meaningful parameters. We will focus our discussion on two systems: the dynamic $\Phi^{4}$ equation and parabolic Anderson model. For the KPZ equation, we save this discussion until Section 6, where we will also highlight the notion of universality.

5.1. $\Phi^{4}$ equation. Let us consider the example of a magnet near its critical temperature in Section 3. Many mathematical models have been proposed to describe various behaviors of magnetic systems. Here we investigate one particular example called the Kac-Ising model 25

We define the model in two dimensions. Denote by $\Lambda_{N}=\mathbf{Z}^{d} /(2 N+1) \mathbf{Z}^{d}$ a large two-dimensional discrete torus of "radius" $N=\varepsilon^{-1}$ (we introduce $\varepsilon$ here to later take to zero), which represents the space in which our magnetic material lives. Each site $k \in \Lambda_{N}$ is decorated with a spin $\sigma(k)$ which for simplicity is assumed to take values in $\{-1,+1\}$. Denote by $\Sigma_{N}=\{-1,+1\}^{\Lambda_{N}}$ the set of spin configurations on $\Lambda_{N}$. For a spin configuration $\sigma=\left(\sigma(k), k \in \Lambda_{N}\right) \in \Sigma_{N}$, we define the Hamiltonian as

$$
H(\sigma):=-\frac{1}{2} \sum_{k, j \in \Lambda_{N}} \kappa_{\gamma}(k-j) \sigma(j) \sigma(k),
$$

where, for $\gamma \in(0,1), \kappa_{\gamma}(k)$ is a nonnegative function 26 supported on $|k| \leq \gamma^{-1}$ which integrates to 1 . Then for any inverse temperature $\beta>0$ we can define the Gibbs measure $\lambda_{\gamma}$ on $\sigma \in \Sigma_{N}$ as

$$
\lambda_{\gamma}(\sigma):=\frac{1}{Z(\beta)} \exp (-\beta H(\sigma))
$$

where $Z(\beta):=\sum_{\sigma \in \Sigma_{N}} \exp (-\beta H(\sigma))$ makes $\lambda_{\gamma}$ into a probability measure.

The measure $\lambda_{\gamma}$ is known as an equilibrium measure since the probability of finding a configuration $\sigma$ is proportional to the exponential of the energy of that configuration. It is also known as equilibrium because it arises as the equilibrium (or stationary, or invariant) measure for various simple, local stochastic dynamics on the configuration space. We will consider one such example known as Glauber dynamics Gla63. For $j \in \Lambda_{N}$, let $\sigma^{j} \in \Sigma_{N}$ denote the spin configuration that coincides with $\sigma$ except the spin at position $j$ is flipped: $\sigma^{j}(j)=-\sigma(j)$. The Glauber dynamic is the following continuous time Markov process: For each $j \in \Lambda_{N}$,

\footnotetext{
${ }^{25}$ The Ising model was introduced in 1920 by Lenz and named after his student Ising who showed that in one spatial dimension it did not admit any phase transition. That original model involves nearest-neighbor pair interactions of -1 and +1 spins. There are many generalizations of this model besides the long-range Kac-Ising model we consider here. For instance, one can consider the higher spin versions with $q$ different types of spins and interactions which award equal spins along edges. This is known as the $q$-state Potts model.

${ }^{26}$ A concrete choice of the interaction kernel $\kappa_{\gamma}$ is to set $\kappa_{\gamma}(k)=c_{\gamma} \gamma^{d} \mathfrak{R}(\gamma k)$, where $\mathfrak{R}: \mathbf{R}^{d} \rightarrow$ $\mathbf{R}$ is a smooth, nonnegative function with compact support and $c_{\gamma}$ is chosen to ensure that $\sum_{k \in \Lambda_{N}} \kappa_{\gamma}(k)=1$.
} 


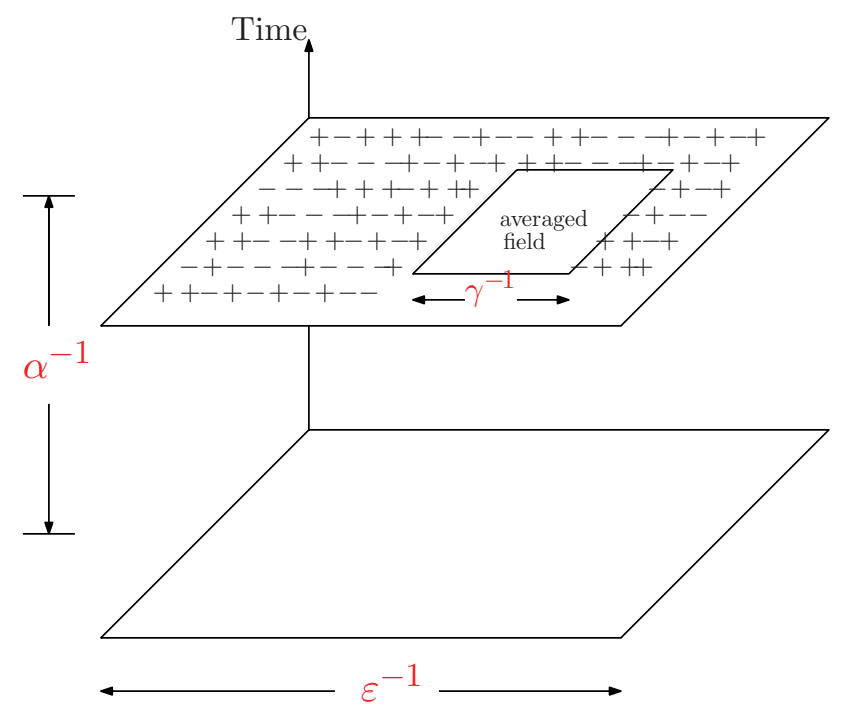

FiguRE 5.1

the configuration $\sigma$ is updated to $\sigma^{j}$ at rate $27 c_{\gamma}(\sigma, j)$ where

$$
c_{\gamma}(\sigma, j):=\frac{\lambda_{\gamma}\left(\sigma^{j}\right)}{\lambda_{\gamma}(\sigma)+\lambda_{\gamma}\left(\sigma^{j}\right)} .
$$

Once an update occurs for some $j$, all rates are recalculated relative to the new configuration. It is standard to show that the measure $\lambda_{\gamma}$ is the unique invariant measure for the Glauber dynamic, meaning that for any starting measuring, eventually the measure will converge in distribution to $\lambda_{\gamma}$. Likewise, if started according to $\lambda_{\gamma}$, then the distribution at any later time will still be distributed according to that measure.

Figure 5.1 illustrates the dynamics where, for each fixed time $t$, one has a spin configuration $\sigma(t)$. We would like to observe a scaling limit of the system from a large distance scale of $\varepsilon^{-1}$ and a long time scale of $\alpha^{-1}$. At larger scales, the $\sigma$ oscillating between \pm 1 would yield a field in a very weak topology; it will be more convenient to consider an averaged field 28

$$
h_{\gamma}(\sigma(t), k):=\sum_{\ell \in \Lambda_{N}} \kappa_{\gamma}(k-\ell) \sigma(t, \ell) .
$$

\footnotetext{
${ }^{27}$ For those not used to continuous time Markov processes, let us explain this more precisely. Starting from some configuration $\sigma$, to each $j \in \Lambda_{N}$ we associate independent exponentially distributed random variables of rate $c_{\gamma}(\sigma, j)$ (i.e., with mean inverse to the rate). One then compares all of these random variables, and for the $j$ whose random variable is minimal, the configuration updates to $\sigma^{j}$. The time at which this occurs is the value of the associated random variable. From that point on, one repeats the whole story, choosing new exponential random variables with rates given by the updated $\sigma^{j}$. Due to the "memoryless" property of exponential random variables (i.e., for $X$ exponential of rate 1 , conditional on $X>x$, the law of $X-x$ is that of a rate 1 exponential random variable), this constructs a continuous time Markov process.

${ }^{28}$ Using the interaction kernel $\kappa_{\gamma}$ to average out the field $\sigma$ is merely a matter of convenience, and it will lead to a clean form of (5.5). Also, convergence of $\sigma$ follows a fortiori.
} 
We may also abuse notation and write $h_{\gamma}(t, k)=h_{\gamma}(\sigma(t), k)$, suppressing the explicit dependence on $\sigma(t)$.

Remark 5.1. In order to prove an SPDE limit, we first write down a discretized SPDE. Generally, this involves a coupled system of stochastic differential equations driven by martingales (recall the brief discussion from Section 4.31). Without delving deeply into details of the theory of Markov processes, let us illustrate this with a simple example. Our applications of this general idea will be more involved, though we will avoid going into details there also. Consider a continuous time random walk $x(t)$ on $\mathbf{Z}$ where a left jump (by 1 ) occurs at rate $\ell$ and a right jump (by 1 ) occurs at rate $r$. For any function $f: \mathbf{Z} \rightarrow \mathbf{R}$, the expected value $u(t, y)=\mathbf{E}_{y}[f(x(t))]$ (where $\mathbf{E}_{y}$ means the expected value assuming initial data $x(0)=y$ ) satisfies the system of ODEs $\frac{\partial}{\partial t} u(t, y)=L u(t, y)$, where the operator $L$ acts on functions $g(y)$ as $(L g)(y):=\ell(g(y-1)-g(y))+r(g(y+1)-g(y))$. Without taking the expectations, $f(x(t))$ will satisfy the evolution equation

$$
d f(x(t))=(L f)(x(t)) d t+d m(t),
$$

where the first term is a drift and the extra term $d m(t)$ is the time derivative of a martingale. The martingale $m(t)$ can be explicitly described in terms of what are called compensated Poisson processes or, equivalently, in terms of its quadratic variation. Under the diffusive scaling which takes $x(t)$ to a Brownian motion, the martingale converges to the time derivative of Brownian motion (what we called one-dimensional white noise earlier in Section 4.3). This can be shown, for example, via the martingale problem for Brownian motion. For more complicated Markov processes, the story is analogous, albeit the analogues of $L$ (known as the instantaneous generator) and $m(t)$ are more complicated.

There are three steps to finding an SPDE limit for the Glauber dynamic on the Kac-Ising model.

5.1.1. Microscopic evolution. In the spirit of Remark 5.1 we may write down the evolution the Markov process $h_{\gamma}$ (5.3) in terms of a drift part (that we make explicit) and a martingale part $m$ (that we do not precisely describe below):

$$
d h_{\gamma}(\sigma(t), x)=\sum_{j \in \Lambda_{N}} c_{\gamma}(\sigma(t), j)\left(h_{\gamma}\left(\sigma^{j}(t), x\right)-h_{\gamma}(\sigma(t), x)\right) d t+d m(\sigma(t), x) .
$$

Recall that $c_{\gamma}$ is given in (5.2). We may look for scaling so that $X_{\gamma}(t, x):=$ $\frac{1}{\delta} h_{\gamma}\left(\sigma\left(\alpha^{-1} t\right), \varepsilon^{-1} x\right)$ converges to a limiting (nonlinear) SPDE. After Taylor expanding the nonlinear dynamic (5.4) into polynomials in $h_{\gamma}$ and passing to $X_{\gamma}$, we get the discrete equation

$$
d X_{\gamma}=\left(\frac{\varepsilon^{2}}{\gamma^{2}} \frac{1}{\alpha} \Delta_{\gamma} X_{\gamma}+\frac{\beta-1}{\alpha} X_{\gamma}-\frac{\delta^{2}}{\alpha} X^{3}+\cdots\right) d t+d M .
$$

Here $\Delta_{\gamma}$ is a difference operator (based on the kernel $\kappa_{\gamma}$ ) which is approximately the Laplacian. The martingale $M$ is a rescaled version of $m$, whose quadratic variation can be also explicitly calculated. We omit this, though we note that one should think of the noise term $d M$ as being of order $O\left(\frac{\varepsilon}{\delta \sqrt{\alpha}}\right)$, in the sense that the quadratic variation of $M$ is of order $O\left(\frac{\varepsilon^{2}}{\delta^{2} \alpha}\right)$. 
5.1.2. Scaling. We may now seek suitable choices for $\varepsilon, \alpha, \gamma, \delta$ such that the dynamics converge to a limit (the $\Phi^{4}$ equation). In particular, we choose $\varepsilon \approx \gamma^{2}, \alpha \approx \gamma^{2}$, and $\delta \approx \gamma$ so that the Laplacian, cubic, and martingale terms all balance:

$$
d X_{\gamma}=(\overbrace{\frac{\varepsilon^{2}}{\gamma^{2}} \frac{1}{\alpha}}^{O(1)} \Delta_{\gamma} X_{\gamma}+\overbrace{\frac{\beta-1}{\alpha}}^{\text {tune } \beta \approx \beta_{c}}=1 \quad X_{\gamma}-\overbrace{\frac{\delta^{2}}{\alpha}}^{O(1)} X_{\gamma}^{3}+\cdots) d t+\overbrace{d M}^{O(1)} .
$$

The critical inverse temperature (at which the magnet loses magnetization) $\beta_{c}=1$ is precisely the value such that sending $\beta \rightarrow \beta_{c}$ at a suitable rate will suppress the linear term from blowing up. Tuning $\beta=1+o(\alpha)$, at large scales (i.e., $\gamma \searrow 0$ with $\varepsilon, \alpha, \delta$ as above) one would expect that $X_{\gamma}$ converges to the solution to the equation 29

$$
\partial_{t} \Phi=\Delta \Phi-\Phi^{3}+\xi
$$

This, however, is not the case. $X_{\gamma}^{3}$ only admits a nontrivial limit when suitably renormalized.

5.1.3. Renormalization. Recalling the discussion in Section 4 (in particular equation (4.4) ), the correct way of taking the limit is to subtract a renormalization term $C_{\gamma} X_{\gamma}$ after the cubic term where $C_{\gamma}$ is the suitably divergent constant. We then add this term back in the linear term so that the equation remains unchanged:

$$
d X_{\gamma}=\left(\frac{\varepsilon^{2}}{\gamma^{2}} \frac{1}{\alpha} \Delta X_{\gamma}+\left(\frac{\beta-1}{\alpha}-\frac{\delta^{2} C_{\gamma}}{\alpha}\right) X_{\gamma}-\frac{\delta^{2}}{\alpha}\left(X_{\gamma}^{3}-C_{\gamma} X\right)+\cdots\right) d t+d M .
$$

In two spatial dimensions, $C_{\gamma}$ diverges logarithmically according to the calculation in equation (4.3). Scaling $\varepsilon, \alpha, \gamma, \delta$ as above, but now tuning the inverse temperature in the correct way $\beta=1+\delta^{2} C_{\gamma}+o(\alpha)$ so that $\beta \rightarrow \beta_{c}=1$ but with a slightly modified rate, $X_{\gamma}$ converges to the solution to the renormalized $\Phi^{4}$ equation. This is proved in MW17a 30

We emphasize two important points: First, the model (5.1) is an interpolation between the standard nearest-neighbor Ising model and a mean-field Ising model (also called the Curie-Weiss model), where all sites interact equally with each other. These two extreme cases have rather different behaviors than the Kac-Ising model. For instance, limits of the nearest-neighbor Ising model lead to conformal invariant objects; see, for example, CHI15] and references therein. On the other hand, the mean-field Ising model with interaction length of order $N$ has Gaussian fluctuations for the magnetization. The $\Phi^{4}$ equation arises in an intermediate or cross-over scale where it is possible to balance all desired terms in (5.5).

The second point is that the renormalization constant in this model represents the delicate rate at which the temperature approaches criticality. Instead, if we did not tune $\beta$ to $\beta_{c}$ properly, the (averaged) magnetic field would either become deterministic (when $\beta>\beta_{c}$ ) or completely disordered, like smoothed white noise

\footnotetext{
${ }^{29}$ One might be puzzled how we can obtain this limiting equation which is not scaling invariant (recall Remark 3.1) via a scaling limit procedure. The reason is that the interaction range $\gamma^{-1}$ in the model (5.1) sets an additional scale.

${ }^{30}$ A similar result in one spatial dimension was shown in the 1990s in [Fri87, FR95]. In one or two spatial dimensions the $\Phi^{4}$ equation is not the only possible limit. SW18 considered a generalized ferromagnetic model (called the Kac-Blume-Capel model) and proves that the Glauber dynamic converges to either the $\Phi^{4}$ or $\Phi^{6}$ equations in various regimes. In three spatial dimensions the $\Phi^{4}$ equation is believed to be the only nontrivial SPDE limit one can obtain from ferromagnetic models, though the proof is still open.
} 
(when $\beta<\beta_{c}$ ). It is only in this critical scaling window that we see the balance between these two ordered and disordered phases.

5.2. Parabolic Anderson equation. We turn to study the parabolic Anderson model (PAM), which describes population dynamics.

5.2.1. Microscopic evolution. Consider the discrete PAM for $v: \mathbf{R}_{+} \times \mathbf{Z}^{d} \rightarrow \mathbf{R}$ :

$$
\partial_{t} v(t, x)=\Delta_{\mathrm{d}} v(t, x)+v(t, x) \eta(x) .
$$

Here $\Delta_{\mathrm{d}}$ can be taken as the generator of a general symmetric random walk, but for simplicity we will assume it is the discrete Laplacian on $\mathbf{Z}^{d}$ (i.e., the generator of a simple symmetric random walk). We also assume for simplicity that $\left\{\eta(x): i \in \mathbf{Z}^{d}\right\}$ is family of i.i.d. mean-zero Gaussian random variables (though in general this randomness does not have to be Gaussian distributed).

The PAM (5.6) models random walks which can branch or die in a given random environment $\eta$. These are particles on the lattice $\mathbf{Z}^{d}$ which all independently follow the dynamics of $\Delta_{\mathrm{d}}$-generator random walk, and which at each lattice point $x \in \mathbf{Z}^{d}$ get killed with rate $\eta(x)^{-}=\max (-\eta(x), 0)$ and branch into two particles with rate $\eta(x)^{+}=\max (\eta(x), 0)$; after the branching the two particles follow the same dynamics. All particles evolve, branch, and die independently of each other. The function $v(t, x)$ is the expected number of particles at time $t$ in location $x$, conditioned on the random environment $\eta$. This model is used to study, for example, the population evolution for microorganisms which flourish in regions with high concentrations of nutrition (i.e., $\eta$ large) and perish in regions with little concentrations of nutrition (i.e., $\eta$ small).

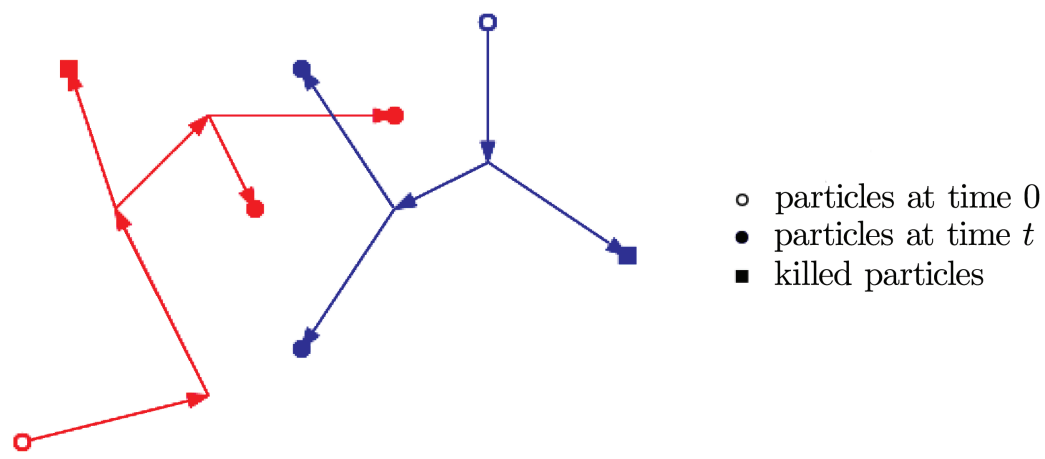

Figure 5.2. An illustration of a situation that starts from two particles and, after branching and dying, ends up with four particles at time $t$.

In what follows we will assume that the dimension $d \leq 3$.

5.2.2. Scaling. Intuitively, $v$ should have high peaks in regions where the environment is most favorable for the particles ( $\eta$ large), and it should have deep valleys elsewhere. After a long time, the bulk of the mass will be concentrated in different scales in these isolated islands, a phenomena known as intermittency 31 Being

\footnotetext{
${ }^{31}$ We refer to the surveys [CM94 and K16] for the intermittency properties of the discrete PAM.
} 
intermittent, we cannot expect to see nontrivial behavior on large spatial scales, since peaks and valleys are microscopic in their nature.

On the other hand, the heat equation (when we set $\eta \equiv 0$ ) smooths particle density. This suggests that in order to obtain a meaningful SPDE scaling limit of the discrete PAM, we ought to tune the strength of the potential $\eta$ so that its influence (and the associated intermittency) balances with the smoothing effect of the heat equation. This should be thought as analogous to the necessary tuning of the magnetic interaction length scale $\gamma^{-1}$ in Section [5.1, or, the weakly asymmetric scaling that arises in KPZ equation convergence in Section 6 below. This kind of tuning of the physical models is generally necessary in order to obtain limiting SPDEs that are not scaling invariant; see Remark 3.1.

Instead of (5.6), let us consider the discrete PAM with weakened noise:

$$
\partial_{t} v(t, x)=\Delta v(t, x)+\varepsilon^{2-\frac{d}{2}} v(t, x) \eta(x), \quad(t, x) \in \mathbf{R}_{+} \times \mathbf{Z}^{d} .
$$

It is reasonable (though false) to believe that the diffusively rescaled solution $v\left(\varepsilon^{-2} t, \varepsilon^{-1} x\right)$ of (5.7) should converge to the solution $w$ of the continuous PAM,

$$
\partial_{t} w(t, x)=\Delta w(t, x)+\sigma w(t, x) \xi(x), \quad(t, x) \in \mathbf{R}_{+} \times \mathbf{R}^{d},
$$

where $\sigma^{2}$ is the variance of $\eta(0)$ and $\xi$ is spatial white noise (i.e., the Gaussian random field with mean zero and covariance $\mathbf{E}[\xi(x) \xi(y)]=\delta(x-y)$ described in Section 2). Note that the choice of weak noise strength $\varepsilon^{2-\frac{d}{2}}, \varepsilon^{2}$ reflects the scaling dimension of the Laplacian operator, and $\varepsilon^{-\frac{d}{2}}$ reflects the scaling dimension of the noise $\xi$ (since the scaling dimension of $\delta$ is $-d$ ).

5.2.3. Renormalization. It turns out that the above naive derivation of (5.8) from (5.7) is not correct (nor is the result true) due to the singular nature of equation (5.8). In fact, even to make sense of equation (5.8) in the continuum setting, one has to introduce some renormalization. In dimensions $d \geq 2$, the total number of particles grows exponentially (even with weak noise) and thus we have to renormalize by this expected growth rate in order to see nontrivial behavior. More precisely, for $t>0$ the expected number of particles at time $\varepsilon^{-2} t$ will be of order $e^{t C_{\varepsilon}}$ with a specific constant $C_{\varepsilon}=O(\log \varepsilon)>0$ in $d=2$ and $C_{\varepsilon}=O\left(\varepsilon^{-1}\right)>0$ in $d=3$. So, one should instead consider

$$
u_{\varepsilon}(t, x):=e^{-t C_{\varepsilon}} v\left(\varepsilon^{-2} t, \varepsilon^{-1} x\right),
$$

which solves the modified and scaled discrete PAM,

$$
\partial_{t} u_{\varepsilon}(t, x)=\Delta^{\varepsilon} u_{\varepsilon}(t, x)+u_{\varepsilon}(t, x)\left(\eta_{\varepsilon}(x)-C_{\varepsilon}\right) .
$$

Here $\Delta^{\varepsilon}$ is a scaling of the discrete Lapacian which approximates the continuous Laplacian as $\varepsilon \rightarrow 0$, and $\eta_{\varepsilon}$ is a scaled version of $\eta$ that converges to $\xi$. This is precisely the form of the renormalized parabolic Anderson equation given by regularity structures in [Hai14b] and paracontrolled distributions in GIP15] if $\eta_{\varepsilon}$ is a mollification of the white noise.

It was rigorously proved in CGP17] that, when $d=2$, the solution $u_{\varepsilon}$ to (5.9) converges in law to the solution of the renormalized (5.8), where the potential $\eta$ is assumed to be a generally distributed (under certain very weak assumptions), and the discrete Laplacian can be generator of any symmetric random walk whose increments have sufficiently many moments. In $d=3$ the same result is expected to hold under such general assumptions on the random walks and random environment. The proof of [CGP17] is based on paracontrolled distributions, introduced in 
Section 4.2. The result [CGP17] is further generalized by MP17] which proves such a convergence result for the nonlinear parabolic Anderson model (3.4) where the factor $f(u)$ models some interaction between the individual particles. The proofs require showing convergence of the perturbative objects discussed in Section 4.2 in the discrete settings, which is one of the main technical challenges; the argument of (3.4) relies on some general tools developed by [CSZ17a].

\section{Singular SPDEs as universal obJeCts}

The singular SPDEs we have studied here are universal objects which arise under various different approximation schemes. It can be shown that continuous mollification in position space as in Hai14b, regularization in Fourier space as in GIP15, and lattice approximations (for instance [GP17b, CM16] for KPZ, [HM18, ZZ18] for $\Phi^{4}$, and references therein) will all lead to the same limiting solution for the SPDEs we discussed in the previous sections. The choices of renormalization constants generally depend on the specific way of approximation in order to obtain the same limit.

Being universal objects means more: each of these singular SPDEs governs the large scale fluctuation of a large class of physical models that have apparently very different microscopic interactions and details. In this section we demonstrate this universality for the KPZ equation by reviewing several recent scaling limit results. We choose to focus on the KPZ equation because, on one hand, there has been quite a lot of progress on KPZ in the last decade, and, on the other hand, several different approaches to solution theories of the KPZ equation have been found. These approaches all yield equivalent notions of solutions to this equation. However, when proving that a convergence results, some notions are better adapted to certain circumstances than others. Our examples will illustrate the application of some of these solution theories.

We start by discussing a continuous formulation of such a universality result proved for the KPZ equation, and then move to more discrete models.

6.1. KPZ equation with general nonlinearity. Consider the KPZ equation with quadratic nonlinear strength $\lambda$,

$$
\partial_{t} H=\partial_{x}^{2} H+\lambda\left(\partial_{x} H\right)^{2}+\xi
$$

and Gaussian space-time white noise $\xi$. This is a widely accepted model for a growing interface subject to three types of local forces: the term $\partial_{x}^{2} H$ models a smoothing mechanism; the term $\lambda\left(\partial_{x} H\right)^{2}$ models lateral growth (the interface tends to grow in the direction normal to the local slope), and $\xi$ models space-time randomness arising on a microscopic scale. In the seminal work of Kardar, Parisi, and Zhang [KPZ86], they justified their eponymous equation by saying that,

the noise has a Gaussian distribution,... although the actual form of the distribution is irrelevant.

They continued to argue that,

growth occurs in a direction locally normal to the interface,.. the increment projected along the $h$ axis is $\delta h=\left[(v \delta t)^{2}+\left(v \delta t \partial_{x} h\right)^{2}\right]^{\frac{1}{2}} \approx$ $\left[v+\frac{v}{2}\left(\partial_{x} h\right)^{2}+\cdots\right] \delta t, \ldots$ the original equation is regained. Such a nonlinear term is clearly expected in all situations where lateral growth is allowed. 
This heuristic derivation of the KPZ equation represents a claim of universality that can be formulated and proved mathematically. Consider the following class of continuous interface growth models where the microscopic growth equation is given by

$$
\partial_{t} h=\partial_{x}^{2} h+\sqrt{\varepsilon} F\left(\partial_{x} h\right)+\eta,
$$

where $F$ is a continuous function modeling the microscopic lateral growth (which could be rather complicated), and $\eta$ is a continuous random field modeling the microscopic randomness (which is generally distributed and not necessarily Gaussian). The coefficient $\sqrt{\varepsilon}$ here corresponds to the weakly asymmetric regime meaning that the small scale interactions are tuned to be small (otherwise they would blow up upon scaling). The challenge is to show that by scaling suitably and applying the correct renormalization, one always obtains the standard KPZ equation (6.1).

Much progress has been achieved thanks to the recent developments of singular SPDE solution theory. The following two results are achieved by the theory of regularity structures.

Hairer and Quastel HQ18] considered the above model (6.2), assuming that $\eta$ is Gaussian but $F$ is an arbitrary even polynomial. They proved that for the rescaled height function defined by $\widetilde{h}_{\varepsilon}(x, t)=\varepsilon^{\frac{1}{2}} h\left(\varepsilon^{-1} x, \varepsilon^{-2} t\right)$ there exist constants $v_{\varepsilon}$ and $\lambda$ such that $\widetilde{h}_{\varepsilon}(x, t)-v_{\varepsilon} t$ converges as $\varepsilon \rightarrow 0$ to the solution to the KPZ equation (6.1) with nonlinearity $\lambda\left(\partial_{x} H\right)^{2}$. This might sound not very surprising because under the above scaling, $\widetilde{h}_{\varepsilon}$ will satisfy a KPZ equation with error terms of the form $\varepsilon^{k}\left(\partial_{x} \widetilde{h}_{\varepsilon}\right)^{2 k+2}$ with $k \geq 1$. However, a very nontrivial fact is that the mean interface growth velocity $v_{\varepsilon}$ (which is a renormalization constant in this context) and the limiting interaction strength $\lambda$ depend explicitly on all coefficients of the polynomial $F$. The essence behind this nontrivial fact is again renormalization: via a similar discussion as in Section 4.1, an error term, such as $\varepsilon\left(\partial_{x} \widetilde{h}_{\varepsilon}\right)^{4}$, needs to be renormalized as

$$
\varepsilon\left[\left(\partial_{x} \widetilde{h}_{\varepsilon}\right)^{4}-C_{\varepsilon}\left(\partial_{x} \widetilde{h}_{\varepsilon}\right)^{2}-\bar{C}_{\varepsilon}\right]
$$

in order to converge to zero; it turns out that $C_{\varepsilon} \sim O\left(\varepsilon^{-1}\right)$, so that $\varepsilon C_{\varepsilon}$ is a finite constant contributing to the limiting coefficient $\lambda$. The constant $\varepsilon \bar{C}_{\varepsilon}$ is still divergent and makes a nontrivial contribution to the velocity $v_{\varepsilon}$. Note that one does not change the model (6.2) in this renormalization procedure; in fact one just reshuffles the polynomial $F$ into linear combinations of renormalized terms like (6.3).

In parallel, HS17] focused on generally distributed $\eta$ in (6.2) with $F$ assumed to be quadratic $F\left(\partial_{x} h\right)=\lambda\left(\partial_{x} h\right)^{2}$. Under a very weak mixing assumption on the non-Gaussian field $\eta$ (so that $\eta_{\varepsilon}(t, x)=\varepsilon^{-\frac{3}{2}} \eta\left(\frac{t}{\varepsilon^{2}}, \frac{x}{\varepsilon}\right)$ converges to the Gaussian white noise $\xi$ by the classical central limit theorem), they demonstrated that with the correct choices of velocities $v^{x}$ and $v_{\varepsilon}^{y}$,

$$
\widetilde{h}_{\varepsilon}\left(x-v^{x} t, t\right)-v_{\varepsilon}^{y} t
$$

converges in law to the same solution to KPZ (6.1) driven by Gaussian white noise $\xi$. It is important to note that the convergence of noise $\eta_{\varepsilon} \rightarrow \xi$ does not imply convergence of solutions. In fact, this is the whole point of building the perturbative random objects in Sections 4.1 and 4.2. Interestingly, the velocity $v^{x}$ in the Galilean transformation shows up since the covariance of $\eta$ is generally not symmetric under 
spatial reflection $x \mapsto-x$; and the mean velocity $v_{(\varepsilon)}^{y} \sim \varepsilon^{-1} C_{0}+\varepsilon^{-\frac{1}{2}} C_{1}+C_{2}$, where $C_{1}$ and $C_{2}$ depend on the third and fourth cumulant 32 of $\eta$, respectively. The velocities $v^{x}$ and $v_{\varepsilon}^{y}$ are renormalization constants which, as in our discussion in Section 4, have explicit expressions in terms of the moments or cumulants of $\eta$ and heat kernels.

These two results HQ18 and [HS17 to a large extent justify the heuristic derivation performed in KPZ86 of the KPZ equation. In addition, they showed that the microscopic details - such as higher order polynomial interactions and higher order cumulants in the microscopic randomness - can contribute to the limiting coefficients or the reference frame in which the limit is observed, even when these details seem to just vanish by scaling.

Proving universality theorems in this continuum setting remains an active direction. More recently, $\mathrm{HX18b}$ ] generalized the result of HQ18 by allowing the more general function $F$ (not necessarily polynomial); they essentially only need to assume certain decay of the local distributional norm of the Fourier transform of $F$. In particular, it includes the case $F(u)=\sqrt{1+u^{2}}$ as originally considered in KPZ86. Under a strong assumption that the system is put into the equilibrium state, GP16] provided a very simple proof using the notion of energy solutions (which is a weak solution as mentioned in Section 4.3).

Remark 6.1. One can ask the same universality questions for the so-called phase coexistence models (or reaction-diffusion models) in three spatial dimensions which have the dynamical $\Phi^{4}$ equation as a universal limit. Essentially, this is a continuous version of the model discussed in (5.5), just like the problem (6.2) can be viewed as continuous version of the models we will discuss in the following subsections. We refer to HX18a, where polynomial nonlinearities are treated, and to FG17. where assumptions on nonlinearities are generalized to $C^{9}$ functions. In the case of polynomial nonlinearities, the noise in the phase coexistence models can be nonGaussian SX16, but the case of general function nonlinearity with non-Gaussian noise is still open. Note that the dynamical $\Phi^{4}$ equation in two spatial dimensions does not have this universal property, because in two spatial dimensions the equation with any polynomial nonlinearity is subcritical in the sense of Remark 4.2, while in three spatial dimensions, among the class of nonlinearities that are odd under $\Phi \rightarrow-\Phi$, only the cubic nonlinearity is subcritical.

6.2. The solid-on-solid growth model. In the previous subsection we saw how the KPZ equation arises as a universal limit of a large class of continuous interface growth models. Another direction which has received a lot of interest is in studying how the KPZ equation arises as a scaling limit of discrete growth models. A particularly simple and well-studied one-dimensional interface growth model is the solid-on-solid interface growth model, which is also called the corner growth model or asymmetric simple exclusion process height process. The interface here is an integer-valued function $h(t, x)$ which depends on the continuous time variable $t \in \mathbf{R}_{+}$and the discrete spatial variable $x \in \mathbf{Z}$, subject to the restriction that $|h(x+1)-h(x)|=1$ for all $x$.

The height function $h$ evolves according to a simple Markov process. As shown in Figure 6.1, a $\wedge$-shaped corner is flipped down by 2 into a $\vee$-shaped corner at

\footnotetext{
${ }^{32}$ Gaussian random variables have zero cumulants of order three or higher. Therefore the third and fourth cumulants of $\eta$ represent some of the non-Gaussian bits of $\eta$.
} 


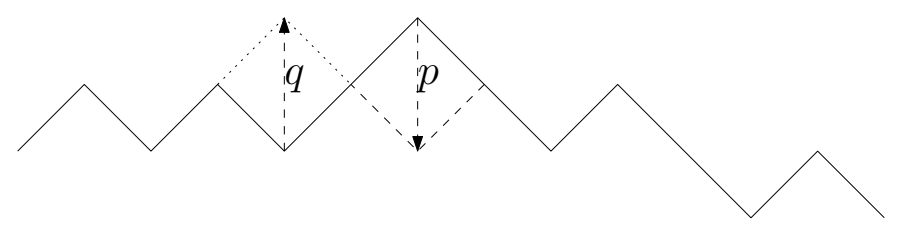

Figure 6.1. A typical plot of a portion of $h(t, \cdot)$ at a given time $t$

rate $p$; and a $\vee$-shaped corner is randomly flipped up by 2 into a $\wedge$-shaped corner at rate $q$. These flips occur independently and according to exponential waiting times (just as described in Section 5.1 for the Kac-Ising model Glauber dynamics). Analogously to the discussion in Remark [5.1, we may derive a microscopic evolution equation for $h$,

$$
d h(t, x)=2\left(q \cdot \mathbf{1}_{\Delta h(t, x)>0}-p \cdot \mathbf{1}_{\Delta h(t, x)<0}\right) d t+d m(t, x),
$$

where $m$ is an explicit martingale. Denoting $\nabla f(x):=f(x+1)-f(x)$ and using our assumption $|\nabla h(x)|=1$, 6.5 can be written into the form of a discrete KPZ type equation,

$$
d h(t, x)=\frac{p+q}{2} \Delta h d t+\frac{q-p}{2}(1-\nabla h(t, x-1) \nabla h(t, x)) d t+d m(t, x) .
$$

In principle, one could implement the theories discussed in the previous sections, such as regularity structures, to prove that for a suitable choice of $v_{\varepsilon}$, $\varepsilon^{\frac{1}{2}} h\left(\varepsilon^{-1} x, \varepsilon^{-2} t\right)-v_{\varepsilon} t$ converges to the solution of the KPZ equation. The main technical challenge in proving this convergence will be showing convergence of the perturbative objects (as described in the case of $\Phi^{4}$ equation in Section 4.2), which are objects built from the discrete heat kernel and the martingale noise $d m$. The martingale noise $d m$ is difficult to deal with since it depends nontrivially on $h$, and there are a fairly large number of such objects that need to be handled. Works such as MW17a, SW18, Mat18 have made progress in studying convergence of approximate SPDEs driven by martingales, but a complete treatment for the KPZ equation is still under way.

Here we present a short-cut approach due to BG97. which applies well to the $\mathrm{KPZ}$ equation and a certain approximation thereof. It is not, however applicable to general SPDEs. Recall from (3.3) that if $u$ solves the stochastic heat equation (SHE)

$$
\partial_{t} u=\frac{1}{2} \Delta u+u \xi
$$

then $H=\log u$ solves the KPZ equation. This defines the Hopf-Cole solution and agrees with the other solution theories defined much later. The Hopf-Cole transform makes rigorous sense as pointed out by [BC95] since the SHE is welldefined in a classical Itô sense Wal86 and since [Mue91 proved strict positivity of $u$ (given rather general initial data). If we can find a version of the Hopf-Cole transform at a microscopic level (i.e., for the solid-on-solid model) and derive an approximate SHE for the exponentiated height function, we can just work at the level of the SHE. 
Gär88,BG97] introduced 33 the Gärtner (or microscopic Hopf-Cole) transform:

$$
Z(t, x):=(p / q)^{\frac{1}{2} h(t, x)} e^{t(1-2 \sqrt{p q})} .
$$

As in Section 5 the evolution of $Z$ can be decomposed into a drift part and a martingale. The drift part turns out to precisely match a discrete Laplacian, namely

$$
d Z(t, x)=\sqrt{p q} \Delta Z(t, x) d t+d M(t, x),
$$

with $M$ a martingale. This is rather surprising since the change $h(t, x) \mapsto h(t, x) \pm 2$ during time $d t$ would seem to affect $Z$ in a nonlinear way. The fact that it holds crucially relies on the fact that $\nabla h$ only takes two possible values $\{ \pm 1\}$. In fact, for more general discrete growth processes, this miracl 34 is generally lost and the approach through the SHE is stymied (see, however, the work of [DT16] discussed further below).

BG97 proved that with $p / q=e^{-\sqrt{\varepsilon}}$ (i.e., weakly asymmetric), $Z\left(\varepsilon^{-2} t, \varepsilon^{-1} x\right)$ converges to the solution to the SHE. Under this scaling,

$$
Z\left(\varepsilon^{-2} t, \varepsilon^{-1} x\right) \approx e^{-\frac{1}{2} \sqrt{\varepsilon} h\left(\varepsilon^{-2} t, \varepsilon^{-1} x\right)-O\left(\varepsilon^{-1}\right) t},
$$

so that the exponent proportional to $t$ in (6.8) is a vertical shift being subtracted as a renormalization.

The proof in BG97. relies on the notion of weak solution (or martingale problems) discussed in Section 4.3 (see, in particular, the discussion around equation (4.14)). In the present context, one only needs to prove that the discrete analogues of the processes $N_{t}$ and $Q_{t}$ in equation (4.14) are approximately martingales. Indeed the fact that the discrete analogue of the process $N_{t}$ is martingale can be immediately read off from (6.9). Showing that the discrete analogue of $Q_{t}$ is approximately martingale amounts to arguing that the quadratic variation of $d M$ in Section 6.9 is approximately the quadratic variation of the limiting term $u \xi$, that is, $u^{2}$. An explicit calculation shows that the quadratic variation involves a term which is approximately $u^{2}$ plus an error term which does not vanish pointwise when passing to the limit. The most challenging part in the proof is to show that this error term vanishes in a suitable averaged sense; we refer to BG97, Eq. (3.16) and Sect. 4.2 "Key estimate"] or [CGST18, Appendix A] for further details.

Remark 6.2. The solid-on-solid interface growth model is equivalent to the asymmetric simple exclusion process which is a paradigmatic model for an interacting particle system. Particles occupy sites indexed by $\mathbf{Z}$ with the restriction of at most one particle per site (indicated by an occupation function at $x \in \mathbf{Z}$ of $\eta(x)=1$ for a particle or $\eta(x)=-1$ for a hole). Each particle attempts to jump left or right according to exponential rates $q$ and $p$, though it only takes the jump if the destination site is not occupied at that time. The occupation process $\eta(t, x)$ coincides with the discrete derivative of the solid so that $\eta(t, x)=\nabla h(t, x)$ (where $\nabla$ acts in the $x$ variable). Due to this, the result of [BG97] also shows that the fluctuation of $\eta$ converges (in a suitable sense) to the solution to the stochastic Burgers equation (4.15) (which arises as the spatial derivative of the KPZ equation).

\footnotetext{
${ }^{33}$ With a slight tweak of notation adapted to our convention.

${ }^{34}$ There is, in fact, a broader class of discrete models which enjoy a version of this exact microscopic transform. These are models which enjoy (at least one particle) Markov duality with respect to exponential functions of the height. See CP16 for a general class of systems which

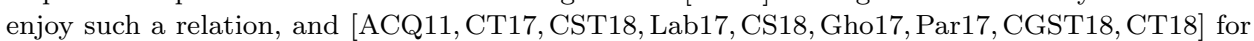
examples of applications of this Markov duality relation to KPZ equation convergence.
} 


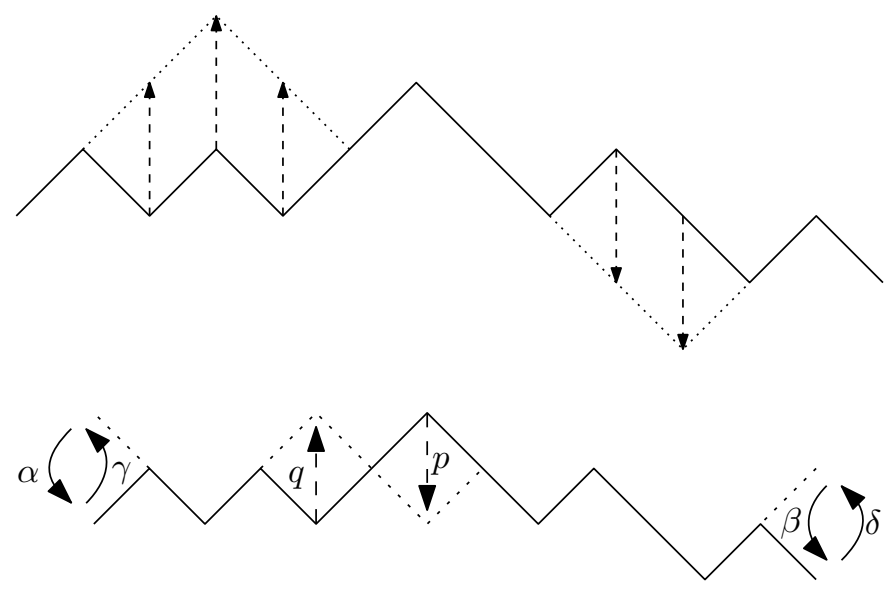

FiguRe 6.2

The work DT16] generalized the result of BG97] by allowing growth or recession of a section (of length at most 4) of the interface; see the top of Figure 6.2. In terms of an interacting particle system, this corresponds with allowing jumps left and right further than distance 1 . The challenge is that the exact match with a discrete Laplacian as in (6.9) is no longer available and instead hydrodynamic limit techniques are used to control the defect in this matching.

Another direction in which [BG97] has been generalized in [CS18, Par17] is to growth models on finite intervals with open boundaries (see the bottom of Figure 6.2). This means that within the interval, the growth rule is as usual, but at the two ends, the height function randomly flips up or down with rates $\alpha, \beta, \gamma, \delta$. With a fine tuning of these parameter 35 it is proved that $\varepsilon^{\frac{1}{2}} h\left(\varepsilon^{-1} x, \varepsilon^{-2} t\right)-C_{\varepsilon} t$ (where $C_{\varepsilon} t$ is the same renormalization as the infinite interface case) converges to $H$ which is the solution of the KPZ equation on the spatial interval $[0,1]$, with inhomogeneous Neumann boundary condition $\left.\partial_{x} H(t, x)\right|_{x=0}=A$ and $\left.\partial_{x} H(t, x)\right|_{x=1}=B$. These two boundary conditions are only formal because $\partial_{x} H$ is distribution and some care is needed to properly define this solution 36

6.3. Six vertex model. The six vertex model $(6 \mathrm{~V})$, originally introduced by [Pau35, is generally defined on a finite box in the two-dimensional square lattice. Each lattice site is occupied by a vertex of one of the six types, with the restriction that vertices join up in a coherent manner (as shown in the following picture) and respect given boundary conditions. Each vertex type has a weight parameter (so in general the model has six parameters), and the probability of a configuration is proportional to the product of all the vertex weights. The height function associated

${ }^{35}$ As in Remark 6.2 there is an underlying interacting particle system called the OpenASEP, which is equivalent to the discrete derivative of this growth model. The OpenASEP exhibits three phases: high density, low density, and maximal current depending on the choices of rate parameters $\alpha, \beta, \gamma, \delta$. The limiting SPDE arises when the parameters are tuned to approach a triple critical point separating these three phases.

36 CS18] defines it via the Hopf-Cole transform of the SHE with Robin boundary condition, which can be defined in the style discussed in Section 4.3. GH17a studied this type of equation with boundary condition from the perspective or regularity structures, and [GPS17] via energy solutions. 


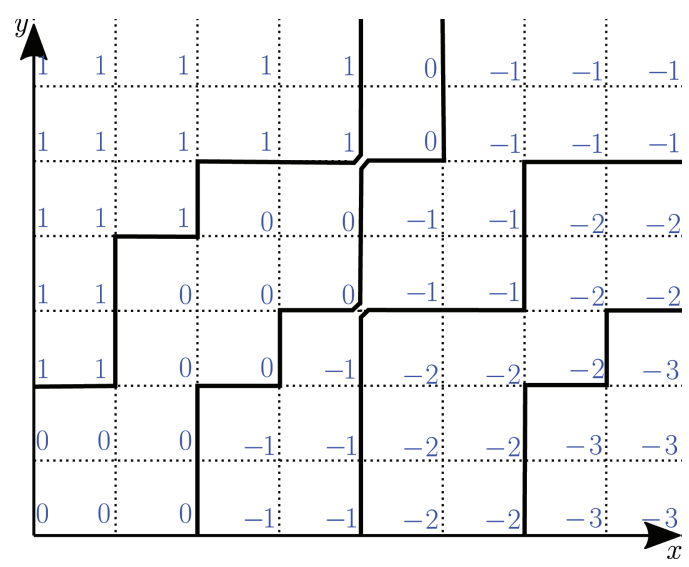

Figure 6.3

with a configuration is the $\mathbf{Z}$-valued function, denoted by $N(x, y)$, that changes by 1 when crossing a line, as shown by the numbers Figure 6.3.

Two special situations are of particular interest: the stochastic $6 \mathrm{~V}$ model and the symmetric $6 \mathrm{~V}$ model. Their vertex weights are given in Table 6.1 .

TABLE 6.1

\begin{tabular}{|c|c|c|c|c|c|c|}
\hline Vertex type & $\vdots$ & $\mathbf{J}$ & $\cdots$ & $\mathbf{|}$ & $\mathbf{\vdots}$ & $\mathbf{J}$ \\
\hline Stochastic $6 V$ weights & 1 & 1 & $b_{1}$ & $b_{2}$ & $1-b_{1}$ & $1-b_{2}$ \\
\hline Symmetric $6 V$ weights & $a$ & $a$ & $b$ & $b$ & $c$ & $c$ \\
\hline
\end{tabular}

The stochastic $6 \mathrm{~V}$ model, proposed by Gwa and Spohn GS92] in 1992, depends on two parameters $b_{1}, b_{2} \in(0,1)$, as shown in Table 6.1. This choice is special since if we treat the bottom and left edges coming into vertices as inputs and the top and right edges as outputs, then the sum of weights over all outputs always equals 1 . This enables us to define the model on the entire first quadrant through a Markovian update. Essentially, the lines can be thought of as paths taken by particles, and the vertex weights determine the probabilities associated to the local moves made by each particle. It is, in fact, also possible to define this particle system on the full upper half-plane, in which case it is natural to think of the $x$-axis as space and the $y$-axis as time (so we use $t$ instead of $y$ to label this direction).

In CGST18 it was proved that under a suitable weak asymmetry, where $b_{2} \rightarrow$ $b_{1}$, upon scaling and renormalizing, the fluctuation of the height function $N(t, x)$ converges to the solution of the KPZ equation. More precisely, fixing any density $\rho \in(0,1)$ of lines entering from the horizontal axis, $b_{1} \in(0,1)$, and $b_{2}=b_{1} e^{-\sqrt{\varepsilon}}$,

$$
\sqrt{\varepsilon}\left(N\left(\varepsilon^{-2} t, \varepsilon^{-1} x+\mu \varepsilon^{-2} t\right)-\rho\left(\varepsilon^{-1} x+\mu \varepsilon^{-2} t\right)\right)-\varepsilon^{-2} t \log \lambda \Longrightarrow H(t, x)
$$

in the topology $C\left(\mathbf{R}_{+}, C(\mathbf{R})\right)$, for some constants $\mu$ and $\lambda$, where $H$ is the solution to the KPZ equation with more general coefficients

$$
\partial_{t} H=\frac{\nu}{2} \partial_{x}^{2} H-\frac{\kappa}{2}\left(\partial_{x} H\right)^{2}+\sqrt{D} \xi .
$$


Here the equation coefficients $\nu=\frac{2 b_{1}}{1-b_{1}}, \kappa=\frac{2 b_{1}}{1-b_{1}}$, and $D=\frac{2 b_{1} \rho(1-\rho)}{1-b_{1}}$. The $\lambda$ and $\mu$ required in the convergence (6.10) also depend on $b_{1}, b_{2}, \rho$ via explicit formulae, which behave like $\lambda=1-\rho \sqrt{\varepsilon}+\mathcal{O}(\varepsilon)$ and $\mu=1+\frac{b_{1}-2 b_{1} \rho}{b_{1}-1} \sqrt{\varepsilon}+\mathcal{O}(\varepsilon)$.

Formula (6.10) shows that in order to observe the KPZ equation fluctuation, one needs to follow a characteristic direction $\mu$, then tilt the height function by $\varepsilon^{-1} \rho x$ (since by definition the function $N$ was tilted), and finally center the height function by subtracting an overall growth speed multiplied by $t$ (i.e., the terms proportional to $t$ in (6.10) ).

The proof of this result relies upon the Hopf-Cole solution, as in Section 6.2, namely, for the KPZ equation as in (6.11), $H=-\frac{\nu}{\kappa} \log u$, where $u$ solves the stochastic heat equation with multiplicative noise as introduced in (3.3),

$$
\partial_{t} u=\frac{\nu}{2} \partial_{x}^{2} u+\frac{\kappa \sqrt{D}}{\nu} \xi u .
$$

Remarkably (and owing to the one-particle duality from [CP16]), just as for the solid-on-solid model in Section 6.2, the stochastic 6V model admits an exact microscopic analogue of this Hopf-Cole transform, transforming it into a discrete version of (6.12). The proof that this discrete version of 6.12) converges to the limiting continuous one requires control over the martingale quadratic variation. The method of [BG97] discussed in Section 6.2 does not seem to apply here, and CGST18 had to introduce a new method relying upon the one- and two-particle duality enjoyed by this model.

Turning very briefly to the older and very well-studied symmetric $6 \mathrm{~V}$ model, as originally proposed by Pau35] in 1935, it turns out that the KPZ equation (or rather stochastic Burgers equation) also arises here. Among the (widely believed, though not generally proved or constructed) two-parameter family of infinite-volume, translation invariant ergodic Gibbs states, Agg16 (see also earlier observations of BS95] in physics) constructed a one-parameter subfamily of "stochastic Gibbs states" using the stochastic $6 \mathrm{~V}$ model. This construction only applies in the ferroelectric regime for parameters $a, b$, and $c$. Zooming into the ferroelectric-disorder phase transition point, and scaling along characteristic directions in the manner described above, these stochastic Gibbs states are shown in CGST18] to converge to stationary solutions to the stochastic Burgers equation.

Let us mention that there are some other works studying SPDE limits of this or closely related models; see, for example, CT17, BO17] or BG18, ST18] (the latter involves a limiting linear hyperbolic SPDE termed the stochastic telegraph equation).

6.4. Interacting Brownian motions. We recall one more convergence result for interacting Brownian motions, as well as the method of energy solutions used.

Consider a collection of independent standard Brownian motions $\left\{W_{t}(i): i \in \mathbf{Z}\right\}$, and define an interface profile $\phi_{t}: \mathbf{Z} \rightarrow \mathbf{R}$ via

$$
\mathrm{d} \phi_{t}(i)=\left\{p V^{\prime}\left(\phi_{t}(i+1)-\phi_{t}(i)\right)-q V^{\prime}\left(\phi_{t}(i)-\phi_{t}(i-1)\right)\right\} \mathrm{d} t+\mathrm{d} W_{t}(i),
$$

for $(t, i) \in \mathbf{R}_{+} \times \mathbf{Z}$ and $p, q \geq 0$ with $p+q=1$. Here, $V$ is a potential function. When $V$ is a constant potential, $\left\{\phi_{t}(i)\right\}_{i \in \mathbf{Z}}$ is simply a collection of independent Brownian motions.

$\phi_{t}$ may be thought of as a one-dimensional interface separating two phases, and we are interested in the random dynamics of this interface. The case $p=q=1 / 2$ describes a type of balance between the two phases and the interface dynamics have 
no net growth; in this case the system is known as the (one-dimensional) GinzburgLandau $\nabla \phi$ interface model which has been intensely studied; see [Fri87, GPV88, Spo86,Zhu90, CY92].

DGP17] proved that under weak asymmetry

$$
p=(1+\sqrt{\varepsilon}) / 2, \quad q=(1-\sqrt{\varepsilon}) / 2,
$$

the properly scaled fluctuation of $\phi$ converges to the solution to the KPZ equation. This result relies on the use of energy solutions as mentioned in Section 4.3, and has only been proved for stationary initial data. In fact, the slope function (or discrete derivative in $i$ )

$$
u_{t}(i):=\phi_{t}(i)-\phi_{t}(i-1)
$$

admits a one-parameter family of stationary measures parametrized by $\lambda \in \mathbb{R}$ :

$$
\mu_{\lambda}(d u):=\prod_{j=-\infty}^{\infty} p_{\lambda}(u(j)) d u(j), \quad \text { where } p_{\lambda}(u):=Z_{\lambda}^{-1} e^{\lambda u-V(u)},
$$

and $Z_{\lambda}=\int_{\mathbf{R}} e^{\lambda u-V(u)} d u$ is a normalization constant. More precisely, DGP17] proved the following result. Assuming $u_{0}(i)=\phi_{0}(i)-\phi_{0}(i-1)$ has the probability distribution $\mu_{\lambda}$ for a fixed $\lambda \in \mathbb{R}$, and let $\rho^{\prime}(\lambda):=\int_{\mathbf{R}} u p_{\lambda}(u) d u$ be the mean of the coordinates $u(j)$ under $\mu_{\lambda}$. Then, under weak asymmetry (6.14), the scaled and renormalized profile process

$$
\varepsilon^{\frac{1}{2}}\left(\phi\left(\varepsilon^{-2} t,\left\lfloor\varepsilon^{-1} x-c_{\varepsilon} t\right\rfloor\right)\right)-C_{\varepsilon}
$$

converge to the solution to

$$
\partial_{t} H=\frac{1}{2 \sigma_{\lambda}^{2}} \partial_{x}^{2} H-\frac{m_{3, \lambda}}{2 \sigma_{\lambda}^{6}}\left(\partial_{x} H\right)^{2}+\xi,
$$

where $c_{\varepsilon}:=\varepsilon^{-1 / 2} \sigma_{\lambda}^{-2}$, the diverging renormalization constant $C_{\varepsilon}$ is explicit, and where $m_{k, \lambda}$ are the moments of the measure $p_{\lambda}$,

$$
m_{k, \lambda}:=\int_{\mathbf{R}}\left(u-\rho^{\prime}(\lambda)\right)^{k} p_{\lambda}(u) d u, \quad \sigma_{\lambda}^{2}:=m_{2, \lambda} .
$$

Since the energy solution is defined for the stochastic Burgers equation, and the solution to stochastic Burgers equals the spatial derivative of that of the KPZ equation (Remark 6.2), the above result is proved via showing that

$$
\varepsilon^{-1 / 2}\left(u\left(\varepsilon^{-2} t,\left\lfloor\varepsilon^{-1} x-c_{\varepsilon} t\right\rfloor\right)-\rho^{\prime}(\lambda)\right)
$$

converges to the energy solution to the stochastic Burgers equation.

The energy solution method for the KPZ/stochastic Burgers equation convergence was initiated in the work of Jara and Gonçalves GJ10] (cf. Ass13]). Initially this approach only provided tightness, in other words, existence of limit, and it was not known whether energy solutions were unique. Uniqueness (and hence the identification with the Hopf-Cole solution of $\mathrm{KPZ}$ as discussed in the previous sections) was proved in GP17a. This approach has been applied to prove that a wide variety of particle systems converge to the KPZ equation; see [GJ14, GJS15, FGS16, GJ13, GJ17, GPS17. These results all require that the system has an invariant measure (i.e., stationary, or equilibrium measure)-like the measures (6.15) for the model (6.13) above - and that the initial condition is thusly distributed. The method of proof relies heavily on having well-developed hydrodynamic theory estimates available. Quite recently, however, GP18c and Yan18] 
have extended this method to include more general initial data as we mentioned in Section 4.3 .

Remark 6.3. Let us conclude this section with several remarks.

First, it would be interesting to compare the expressions (6.4) in the continuous interface model, (6.10), in the six vertex model, and (6.16) for the interface driven by Brownian motions. The common feature is that in all of these cases, the KPZ equation arises via suitable adjustments of reference frames dictated by the renormalizations in these discrete models.

Also, we have seen that the proof strategies of these convergence results depend on which notion of solution is being chosen in the context. The Hopf-Cole solution to the KPZ equation has the obvious advantage in turning the problem to a linear equation (6.7) and (6.12), as we demonstrated in the solid-on-solid growth model and the six vertex model. Although it has been shown for a number of models one can implement this Hopf-Cole transform at the microscopic level, this relation certainly does not exactly hold in general; it is also a special feature for KPZ that is absent for other nonlinear SPDEs discussed in Section 3 . The energy solution has been applied to proving KPZ/stochastic Burgers equation convergence results of systems which have an equilibrium invariant measure and start from this equilibrium. The recent works GP18c, Yan18 extended the type of initial data, and GP18a] studied a more general class of equations; further extending the scope of applicability of the energy solution to physical models seems to be an interesting direction. The theory of regularity structures and paracontrolled distributions method provide robust solution theories for very large classes of equations, and they yielded universality results in the continuum setting of Section 6.1. Proving such convergence results for discrete physical systems driven by martingale noises in a more systematic way would be another interesting direction. In addition to the results cited above, let us mention [HM18, EH17, Mat18] for some initial progress on discrete regularity structures.

\section{ACKNOWLEDGMENTS}

We are grateful to Weinan E, Massimiliano Gubinelli, Martin Hairer, Konstantin Matetski, Nicolas Perkowski, and Li-Cheng Tsai for providing helpful comments while we completed this survey.

\section{ABout THE AUthors}

Ivan Corwin is professor of mathematics at Columbia University. He works primarily in probability and mathematical physics with special interest in the study of stochastic PDEs and integrable probability.

Hao Shen is assistant professor of mathematics at University of WisconsinMadison. He works primarily in probability and mathematical physics with special interest in the study of stochastic PDEs, interacting particle systems, and quantum field theory.

\section{RefERENCES}

[AC15] R. Allez and K. Chouk, The continuous Anderson Hamiltonian in dimension two, arXiv:1511.02718, 2015. 
[ACQ11] G. Amir, I. Corwin, and J. Quastel, Probability distribution of the free energy of the continuum directed random polymer in $1+1$ dimensions, Comm. Pure Appl. Math. 64 (2011), no. 4, 466-537, DOI 10.1002/cpa.20347. MR2796514

[Agg16] A. Aggarwal, Current fluctuations of the stationary ASEP and six-vertex model, Duke Math. J. 167 (2018), no. 2, 269-384, DOI 10.1215/00127094-2017-0029. MR3754630

[AHR96] S. Albeverio, Z. Haba, and F. Russo, Trivial solutions for a non-linear two-spacedimensional wave equation perturbed by space-time white noise, Stochastics Stochastics Rep. 56 (1996), no. 1-2, 127-160, DOI 10.1080/17442509608834039. MR.1396758

[AK17] S. Albeverio and S. Kusuoka, The invariant measure and the flow associated to the $\phi_{3}^{4}$-quantum field model, arXiv:1711.07108, 2017.

[AR91] S. Albeverio and M. Röckner, Stochastic differential equations in infinite dimensions: solutions via Dirichlet forms, Probab. Theory Related Fields 89 (1991), no. 3, 347386, DOI 10.1007/BF01198791. MR.1113223

[Ass13] S. Assing, A rigorous equation for the Cole-Hopf solution of the conservative KPZ equation, Stoch. Partial Differ. Equ. Anal. Comput. 1 (2013), no. 2, 365-388, DOI 10.1007/s40072-013-0013-3. MR3327511

[BB16] I. Bailleul and F. Bernicot, Heat semigroup and singular PDEs, J. Funct. Anal. 270 (2016), no. 9, 3344-3452, DOI 10.1016/j.jfa.2016.02.012. With an appendix by F. Bernicot and D. Frey. MR.3475460

[BC95] L. Bertini and N. Cancrini, The stochastic heat equation: Feynman-Kac formula and intermittence, J. Statist. Phys. 78 (1995), no. 5-6, 1377-1401, DOI 10.1007/BF02180136. MR 1316109

[BCCH17] Y. Bruned, A. Chandra, I. Chevyrev, and M. Hairer, Renormalising SPDEs in regularity structures, arXiv:1711.10239, 2017.

[BCD11] H. Bahouri, J.-Y. Chemin, and R. Danchin, Fourier analysis and nonlinear partial differential equations, Grundlehren der Mathematischen Wissenschaften [Fundamental Principles of Mathematical Sciences], vol. 343, Springer, Heidelberg, 2011. MR 2768550

[BDH19] I. Bailleul, A. Debussche, and M. Hofmanová, Quasilinear generalized parabolic Anderson model equation, Stoch. Partial Differ. Equ. Anal. Comput. 7 (2019), no. 1, 40-63, DOI 10.1007/s40072-018-0121-1. MR3916262

[Ber70] V. L. Berezinskiŭ, Destruction of long-range order in one-dimensional and twodimensional systems having a continuous symmetry group. I. Classical systems (Russian, with English summary), Ž. Èksper. Teoret. Fiz. 59 (1970), 907-920; English transl., Soviet Physics JETP 32 (1971), 493-500. MR0314399

[BG97] L. Bertini and G. Giacomin, Stochastic Burgers and KPZ equations from particle systems, Comm. Math. Phys. 183 (1997), no. 3, 571-607, DOI 10.1007/s002200050044. MR 1462228

[BG18] A. Borodin and V. Gorin, A stochastic telegraph equation from the six-vertex model, arXiv:1803.09137, 2018.

[BGHZ19] Y. Bruned, F. Gabriel, M. Hairer, and L. Zambotti, Geometric stochastic heat equations, arXiv: 1902.02884, 2019.

[BHZ16] Y. Bruned, M. Hairer, and L. Zambotti, Algebraic renormalisation of regularity structures, Invent. Math. 215 (2019), no. 3, 1039-1156, DOI 10.1007/s00222-018-0841-x. MR.3935036

[BO17] A. Borodin and G. Olshanski, The ASEP and determinantal point processes, Comm. Math. Phys. 353 (2017), no. 2, 853-903, DOI 10.1007/s00220-017-2858-1. MR3649488

[Bou96] J. Bourgain, Invariant measures for the 2D-defocusing nonlinear Schrödinger equation, Comm. Math. Phys. 176 (1996), no. 2, 421-445. MR 1374420

[BP15] A. Borodin and L. Petrov, Integrable probability: stochastic vertex models and symmetric functions, Stochastic processes and random matrices, Oxford Univ. Press, Oxford, 2017, pp. 26-131. MR3728714

[Bru68] S. G. Brush, A history of random processes: I. Brownian movement from Brown to Perrin, Arch. History Exact Sci. 5 (1968), no. 1, 1-36, DOI 10.1007/BF00328110. MR.1554112

[BS95] D. J. Bukman and J. D. Shore, The conical point in the ferroelectric six-vertex model, J. Statist. Phys. 78 (1995), no. 5-6, 1277-1309, DOI 10.1007/BF02180132. MR.1316105

[CC18a] G. Cannizzaro and K. Chouk, Multidimensional SDEs with singular drift and universal construction of the polymer measure with white noise potential, Ann. Probab. 46 (2018), no. 3, 1710-1763, DOI 10.1214/17-AOP1213. MR3785598 
[CC18b] R. Catellier and K. Chouk, Paracontrolled distributions and the 3-dimensional stochastic quantization equation, Ann. Probab. 46 (2018), no. 5, 2621-2679, DOI 10.1214/17-AOP1235. MR3846835

[CD18] S. Chatterjee and A. Dunlap. Constructing a solution of the $(2+1)$-dimensional KPZ equation, arXiv: 1809.00803, 2018.

[CGP17] K. Chouk, J. Gairing, and N. Perkowski, An invariance principle for the twodimensional parabolic Anderson model with small potential, Stoch. Partial Differ. Equ. Anal. Comput. 5 (2017), no. 4, 520-558, DOI 10.1007/s40072-017-0096-3. MR3736653

[CGST18] I. Corwin, P. Ghosal, H. Shen, and L.-C. Tsai, Stochastic PDE limit of the Six Vertex Model, arXiv: 1803.08120, 2018.

[CH16] A. Chandra and M. Hairer, An analytic BPHZ theorem for regularity structures, arXiv:1612.08138, 2016.

[CHI15] D. Chelkak, C. Hongler, and K. Izyurov, Conformal invariance of spin correlations in the planar Ising model, Ann. of Math. (2) 181 (2015), no. 3, 1087-1138, DOI 10.4007/annals.2015.181.3.5. MR.3296821

[CHS18] A. Chandra, M. Hairer, and H. Shen, The dynamical sine-Gordon model in the full subcritical regime, arXiv:1808.02594, 2018.

[CM94] R. A. Carmona and S. A. Molchanov, Parabolic Anderson problem and intermittency, Mem. Amer. Math. Soc. 108 (1994), no. 518, viii+125, DOI 10.1090/memo/0518. MR.1185878

[CM16] G. Cannizzaro and K. Matetski, Space-time discrete KPZ equation, Comm. Math. Phys. 358 (2018), no. 2, 521-588, DOI 10.1007/s00220-018-3089-9. MR.3774431

[Con12] C. Conti, Solitonization of the Anderson localization, Physical Review A, 86 (2012), no. 6,061801 .

[Cor14] I. Corwin, Macdonald processes, quantum integrable systems and the KardarParisi-Zhang universality class, Proceedings of the International Congress of Mathematicians-Seoul 2014. Vol. III, Kyung Moon Sa, Seoul, 2014, pp. 1007-1034. MR.3729062

[CP16] I. Corwin and L. Petrov, Stochastic higher spin vertex models on the line, Comm. Math. Phys. 343 (2016), no. 2,651-700, DOI 10.1007/s00220-015-2479-5. MR3477349

[CR99] R. A. Carmona and B. Rozovskii (eds.), Stochastic partial differential equations: six perspectives, Mathematical Surveys and Monographs, vol. 64, American Mathematical Society, Providence, RI, 1999. MR 1661761

[CS18] I. Corwin and H. Shen, Open ASEP in the weakly asymmetric regime, Comm. Pure Appl. Math. 71 (2018), no. 10, 2065-2128, DOI 10.1002/cpa.21744. MR.3861074

[CST18] I. Corwin, H. Shen, and L.-C. Tsai, $\operatorname{ASEP}(q, j)$ converges to the KPZ equation (English, with English and French summaries), Ann. Inst. Henri Poincaré Probab. Stat. 54 (2018), no. 2, 995-1012, DOI 10.1214/17-AIHP829. MR.3795074

[CSZ17a] F. Caravenna, R. Sun, and N. Zygouras, Polynomial chaos and scaling limits of disordered systems, J. Eur. Math. Soc. (JEMS) 19 (2017), no. 1, 1-65, DOI 10.4171/JEMS/660. MR3584558

[CSZ17b] F. Caravenna, R. Sun, and N. Zygouras, Universality in marginally relevant disordered systems, Ann. Appl. Probab. 27 (2017), no. 5, 3050-3112, DOI 10.1214/17-AAP1276. MR 3719953

[CSZ18] F. Caravenna, R. Sun, and N. Zygouras, The two-dimensional KPZ equation in the entire subcritical regime, arXiv:1812.03911, 2018.

[CT17] I. Corwin and L.-C. Tsai, KPZ equation limit of higher-spin exclusion processes, Ann. Probab. 45 (2017), no. 3, 1771-1798, DOI 10.1214/16-AOP1101. MR3650415

[CT18] I. Corwin and L.-C. Tsai, SPDE limit of weakly inhomogeneous ASEP, arXiv:1806. $09682,2018$.

[CW17] A. Chandra and H. Weber, Stochastic PDEs, regularity structures, and interacting particle systems (English, with English and French summaries), Ann. Fac. Sci. Toulouse Math. (6) 26 (2017), no. 4, 847-909, DOI 10.5802/afst.1555. MR3746645

[CY92] C. C. Chang and H.-T. Yau, Fluctuations of one-dimensional Ginzburg-Landau models in nonequilibrium, Comm. Math. Phys. 145 (1992), no. 2, 209-234. MR.1162798

[Daw72] D. A. Dawson, Stochastic evolution equations, Math. Biosci. 15 (1972), 287-316, DOI 10.1016/0025-5564(72)90039-9. MR0321178

[DGP17] J. Diehl, M. Gubinelli, and N. Perkowski, The Kardar-Parisi-Zhang equation as scaling limit of weakly asymmetric interacting Brownian motions, Comm. Math. Phys. 354 (2017), no. 2, 549-589, DOI 10.1007/s00220-017-2918-6. MR3663617 
[DK90] S. K. Donaldson and P. B. Kronheimer, The geometry of four-manifolds, Oxford Mathematical Monographs, The Clarendon Press, Oxford University Press, New York, 1990. Oxford Science Publications. MR1079726

$\left[\mathrm{DKM}^{+}\right.$09] R. Dalang, D. Khoshnevisan, C. Mueller, D. Nualart, and Y. Xiao, A minicourse on stochastic partial differential equations, Lecture Notes in Mathematics, vol. 1962, Springer-Verlag, Berlin, 2009. Held at the University of Utah, Salt Lake City, UT, May 8-19, 2006; Edited by Khoshnevisan and Firas Rassoul-Agha. MR1500166

[DM17] A. Debussche and J. Martin, Solution to the stochastic Schrodinger equation on the full space, arXiv:1707.06431, 2017.

[DPD02] G. Da Prato and A. Debussche, Two-dimensional Navier-Stokes equations driven by a space-time white noise, J. Funct. Anal. 196 (2002), no. 1, 180-210, DOI 10.1006/jfan.2002.3919. MR.1941997

[DPD03] G. Da Prato and A. Debussche, Strong solutions to the stochastic quantization equations, Ann. Probab. 31 (2003), no. 4, 1900-1916, DOI 10.1214/aop/1068646370. MR.2016604

[DPZ14] G. Da Prato and J. Zabczyk, Stochastic equations in infinite dimensions, 2nd ed., Encyclopedia of Mathematics and its Applications, vol. 152, Cambridge University Press, Cambridge, 2014. MR3236753

[DT16] A. Dembo and L.-C. Tsai, Weakly asymmetric non-simple exclusion process and the Kardar-Parisi-Zhang equation, Comm. Math. Phys. 341 (2016), no. 1, 219-261, DOI 10.1007/s00220-015-2527-1. MR3439226

[DW18] A. Debussche and H. Weber, The Schrödinger equation with spatial white noise potential, Electron. J. Probab. 23 (2018), Paper No. 28, 16, DOI 10.1214/18-EJP143. MR 3785398

[EH17] D. Erhard and M. Hairer, Discretisation of regularity structures, arXiv:1705.02836, 2017.

[Fee14] P. M. N. Feehan, Global existence and convergence of smooth solutions to yang-mills gradient flow over compact four-manifolds, arXiv:1409.1525, 2014.

[FG17] M. Furlan and M. Gubinelli, Weak universality for a class of 3d stochastic reactiondiffusion models, Probab. Theory Related Fields 173 (2019), no. 3-4, 1099-1164, DOI 10.1007/s00440-018-0849-6. MR3936152

[FG19] M. Furlan and M. Gubinelli, Paracontrolled quasilinear SPDEs, Ann. Probab. 47 (2019), no. 2, 1096-1135, DOI 10.1214/18-AOP1280. MR3916943

[FGS16] T. Franco, P. Gonçalves, and M. Simon, Crossover to the stochastic Burgers equation for the WASEP with a slow bond, Comm. Math. Phys. 346 (2016), no. 3, 801-838, DOI 10.1007/s00220-016-2607-x. MR.3537337

[FH14] P. K. Friz and M. Hairer, A course on rough paths, Universitext, Springer, Cham, 2014. With an introduction to regularity structures. MR 3289027

[FH17] T. Funaki and M. Hoshino, A coupled KPZ equation, its two types of approximations and existence of global solutions, J. Funct. Anal. 273 (2017), no. 3, 1165-1204, DOI 10.1016/j.jfa.2017.05.002. MR3653951

[Fla08] F. Flandoli, An introduction to 3D stochastic fluid dynamics, SPDE in hydrodynamic: recent progress and prospects, Lecture Notes in Math., vol. 1942, Springer, Berlin, 2008, pp. 51-150, DOI 10.1007/978-3-540-78493-7_2. MR2459085

[Fle75] W. H. Fleming, Distributed parameter stochastic systems in population biology, Control theory, numerical methods and computer systems modelling (Internat. Sympos., IRIA LABORIA, Rocquencourt, 1974), Springer, Berlin, 1975, pp. 179-191. Lecture Notes in Econom. and Math. Systems, Vol. 107. MR0378124

[FOT11] M. Fukushima, Y. Oshima, and M. Takeda, Dirichlet forms and symmetric Markov processes, Second revised and extended edition, De Gruyter Studies in Mathematics, vol. 19, Walter de Gruyter \& Co., Berlin, 2011. MR2778606

[FR95] J. Fritz and B. Rüdiger, Time dependent critical fluctuations of a one-dimensional local mean field model, Probab. Theory Related Fields 103 (1995), no. 3, 381-407, DOI 10.1007/BF01195480. MR1358083

[Fri87] J. Fritz, On the hydrodynamic limit of a one-dimensional Ginzburg-Landau lattice model. The a priori bounds, J. Statist. Phys. 47 (1987), no. 3-4, 551-572, DOI 10.1007/BF01007526. MR894407

[FS81] J. Fröhlich and T. Spencer, The Kosterlitz-Thouless transition in two-dimensional abelian spin systems and the Coulomb gas, Comm. Math. Phys. 81 (1981), no. 4, 527-602. MR634447 
[Fun83] T. Funaki, Random motion of strings and related stochastic evolution equations, Nagoya Math. J. 89 (1983), 129-193, DOI 10.1017/S0027763000020298. MR692348

[Gär88] J. Gärtner, Convergence towards Burgers' equation and propagation of chaos for weakly asymmetric exclusion processes, Stochastic Process. Appl. 27 (1988), no. 2, 233-260, DOI 10.1016/0304-4149(87)90040-8. MR.931030

$\left[\mathrm{GGF}^{+}{ }^{12}\right]$ N. Ghofraniha, S. Gentilini, V. Folli, E. DelRe, and C. Conti, Shock waves in disordered media, Physical Review Letters, 109 (2012), no. 24, 243902.

[GH17a] M. Gerencsér and M. Hairer, Singular SPDEs in domains with boundaries, Probab. Theory Related Fields 173 (2019), no. 3-4, 697-758, DOI 10.1007/s00440-018-0841-1. MR.3936145

[GH17b] M. Gerencsér and M. Hairer. A solution theory for quasilinear singular SPDEs, Comm. Pure Appl. Math., 2017.

[GH18a] M. Gubinelli and M. Hofmanová, A PDE construction of the Euclidean $\Phi_{3}^{4}$ quantum field theory, arXiv:1810.01700, 2018.

[GH18b] M. Gubinelli and M. Hofmanová, Global solutions to elliptic and parabolic $\phi^{4}$ models in Euclidean space, arXiv:1804.11253, 2018.

[Gho17] P. Ghosal, Hall-Littlewood-PushTASEP and its KPZ limit, arXiv:1701.07308, 2017.

[GIP15] M. Gubinelli, P. Imkeller, and N. Perkowski, Paracontrolled distributions and singular PDEs, Forum Math. Pi 3 (2015), e6, 75, DOI 10.1017/fmp.2015.2. MR3406823

[GJ10] P. Goncalves and M. Jara, Universality of KPZ equation. arXiv:1003.4478, 2010.

[GJ13] M. Gubinelli and M. Jara, Regularization by noise and stochastic Burgers equations, Stoch. Partial Differ. Equ. Anal. Comput. 1 (2013), no. 2, 325-350, DOI 10.1007/s40072-013-0011-5. MR3327509

[GJ14] P. Gonçalves and M. Jara, Nonlinear fluctuations of weakly asymmetric interacting particle systems, Arch. Ration. Mech. Anal. 212 (2014), no. 2, 597-644, DOI 10.1007/s00205-013-0693-x. MR.3176353

[GJ17] P. Gonçalves and M. Jara, Stochastic Burgers equation from long range exclusion interactions, Stochastic Process. Appl. 127 (2017), no. 12, 4029-4052, DOI 10.1016/j.spa.2017.03.022. MR.3718105

[GJS15] P. Gonçalves, M. Jara, and S. Sethuraman, A stochastic Burgers equation from a class of microscopic interactions, Ann. Probab. 43 (2015), no. 1, 286-338, DOI 10.1214/13AOP878. MR3298474

[GKO18a] M. Gubinelli, H. Koch, and T. Oh, Paracontrolled approach to the three-dimensional stochastic nonlinear wave equation with quadratic nonlinearity, arXiv:1811.07808, 2018.

[GKO18b] M. Gubinelli, H. Koch, and T. Oh, Renormalization of the two-dimensional stochastic nonlinear wave equations, Trans. Amer. Math. Soc. 370 (2018), no. 10, 7335-7359, DOI 10.1090/tran/7452. MR3841850

[GKR18] Y. Gu, T. Komorowski, and L. Ryzhik, The Schrödinger equation with spatial white noise: the average wave function, J. Funct. Anal. 274 (2018), no. 7, 2113-2138, DOI 10.1016/j.jfa.2018.01.015. MR3762097

[Gla63] R. J. Glauber, Time-dependent statistics of the Ising model, J. Mathematical Phys. 4 (1963), 294-307, DOI 10.1063/1.1703954. MR0148410

[GP16] M. Gubinelli and N. Perkowski, The Hairer-Quastel universality result in equilibrium, arXiv:1602.02428, 2016.

[GP17a] M. Gubinelli and N. Perkowski, Energy solutions of KPZ are unique, J. Amer. Math. Soc. 31 (2018), no. 2, 427-471, DOI 10.1090/jams/889. MR.3758149

[GP17b] M. Gubinelli and N. Perkowski, KPZ reloaded, Comm. Math. Phys. 349 (2017), no. 1, 165-269, DOI 10.1007/s00220-016-2788-3. MR.3592748

[GP18a] M. Gubinelli and N. Perkowski, The infinitesimal generator of the stochastic Burgers equation, arXiv:1810.12014, 2018.

[GP18b] M. Gubinelli and N. Perkowski, An introduction to singular SPDEs, Stochastic partial differential equations and related fields, Springer Proc. Math. Stat., vol. 229, Springer, Cham, 2018, pp. 69-99. MR.3828162

[GP18c] M. Gubinelli and N. Perkowski, Probabilistic approach to the stochastic Burgers equation, Stochastic partial differential equations and related fields, Springer Proc. Math. Stat., vol. 229, Springer, Cham, 2018, pp. 515-527. MR3828193

[GPS17] P. Goncalves, N. Perkowski, and M. Simon, Derivation of the stochastic Burgers equation with Dirichlet boundary conditions from the WASEP, arXiv:1710.11011, 2017. 
[GPV88] M. Z. Guo, G. C. Papanicolaou, and S. R. S. Varadhan, Nonlinear diffusion limit for a system with nearest neighbor interactions, Comm. Math. Phys. 118 (1988), no. 1, 31-59. MR.954674

[GS92] L.-H. Gwa and H. Spohn, Six-vertex model, roughened surfaces, and an asymmetric spin Hamiltonian, Phys. Rev. Lett. 68 (1992), no. 6, 725-728, DOI 10.1103/PhysRevLett.68.725. MR.1147356

[Gu18] Y. Gu. Gaussian fluctuations of the 2D KPZ equation, arXiv:1812.07467, 2018.

[Gub04] M. Gubinelli, Controlling rough paths, J. Funct. Anal. 216 (2004), no. 1, 86-140, DOI 10.1016/j.jfa.2004.01.002. MR2091358

[Gub18] M. Gubinelli, A panorama of singular SPDEs, in Proc. Int. Cong. of Math., volume 2, pp. 2277-2304, 2018.

[GUZ18] M. Gubinelli, B. E. Ugurcan, and I. Zachhuber, Semilinear evolution equations for the Anderson Hamiltonian in two and three dimensions, arXiv:1807.06825, 2018.

[Hai13] M. Hairer, Solving the KPZ equation, Ann. of Math. (2) 178 (2013), no. 2, 559-664, DOI 10.4007/annals.2013.178.2.4. MR3071506

[Hai14a] M. Hairer, Singular stochastic PDEs, Proceedings of the International Congress of Mathematicians-Seoul 2014. Vol. 1, Kyung Moon Sa, Seoul, 2014, pp. 685-709. MR.3728488

[Hai14b] M. Hairer, A theory of regularity structures, Invent. Math. 198 (2014), no. 2, 269-504, DOI 10.1007/s00222-014-0505-4. MR.3274562

[Hai15a] M. Hairer, Introduction to regularity structures, Braz. J. Probab. Stat. 29 (2015), no. 2, 175-210, DOI 10.1214/14-BJPS241. MR3336866

[Hai15b] M. Hairer, Regularity structures and the dynamical $\phi_{3}^{4}$ model, arXiv:1508.05261, 2015.

[Hai16] M. Hairer, The motion of a random string, arXiv:1605.02192, 2016.

[HL15] M. Hairer and C. Labbé, A simple construction of the continuum parabolic Anderson model on $\mathbf{R}^{2}$, Electron. Commun. Probab. 20 (2015), no. 43, 11, DOI 10.1214/ECP.v20-4038. MR3358965

[HL18] M. Hairer and C. Labbé, Multiplicative stochastic heat equations on the whole space, J. Eur. Math. Soc. (JEMS) 20 (2018), no. 4, 1005-1054, DOI 10.4171/JEMS/781. MR.3779690

[HM18] M. Hairer and K. Matetski, Discretisations of rough stochastic PDEs, Ann. Probab. 46 (2018), no. 3, 1651-1709, DOI 10.1214/17-AOP1212. MR.3785597

[HP15] M. Hairer and É. Pardoux, A Wong-Zakai theorem for stochastic PDEs, J. Math. Soc. Japan 67 (2015), no. 4, 1551-1604, DOI 10.2969/jmsj/06741551. MR3417505

[HQ18] M. Hairer and J. Quastel, A class of growth models rescaling to KPZ, Forum Math. Pi 6 (2018), e3, 112, DOI 10.1017/fmp.2018.2. MR.3877863

[HS16] M. Hairer and H. Shen, The dynamical sine-Gordon model, Comm. Math. Phys. 341 (2016), no. 3, 933-989, DOI 10.1007/s00220-015-2525-3. MR3452276

[HS17] M. Hairer and H. Shen, A central limit theorem for the KPZ equation, Ann. Probab. 45 (2017), no. 6B, 4167-4221, DOI 10.1214/16-AOP1162. MR3737909

[HX18a] M. Hairer and W. Xu, Large-scale behavior of three-dimensional continuous phase coexistence models, Comm. Pure Appl. Math. 71 (2018), no. 4, 688-746, DOI 10.1002/cpa.21738. MR.3772400

[HX18b] M. Hairer and W. Xu, Large-scale limit of interface fluctuation models, arXiv:1802. $08192,2018$.

[Hos18] M. Hoshino, Paracontrolled calculus and Funaki-Quastel approximation for the KPZ equation, Stochastic Process. Appl. 128 (2018), no. 4, 1238-1293, DOI 10.1016/j.spa.2017.07.001. MR.3769661

[Jaf00] A. Jaffe, Constructive quantum field theory, Mathematical physics 2000, Imp. Coll. Press, London, 2000, pp. 111-127, DOI 10.1142/9781848160224_0007. MR.1773042

[Jan97] S. Janson, Gaussian Hilbert spaces, Cambridge Tracts in Mathematics, vol. 129, Cambridge University Press, Cambridge, 1997. MR.1474726

[JLM85] G. Jona-Lasinio and P. K. Mitter, On the stochastic quantization of field theory, Comm. Math. Phys. 101 (1985), no. 3, 409-436. MR815192

[Jos13] J. V. Jos, 40 years of Berezinskii-Kosterlitz-Thouless theory. World Scientific, 2013.

[K16] W. König, The parabolic Anderson model, Pathways in Mathematics, Birkhäuser/Springer, [Cham], 2016. Random walk in random potential. MR 3526112 
[KM17] A. Kupiainen and M. Marcozzi, Renormalization of generalized KPZ equation, J. Stat. Phys. 166 (2017), no. 3-4, 876-902, DOI 10.1007/s10955-016-1636-3. MR3607594

[KPZ86] M. Kardar, G. Parisi, and Y.-C. Zhang, Dynamic scaling of growing interfaces, Phys. Rev. Lett., 56 (1986), 889-892.

[KR82] N. V. Krylov and B. L. Rozovskiū, Stochastic partial differential equations and diffusion processes (Russian), Uspekhi Mat. Nauk 37 (1982), no. 6(228), 75-95. MR683274

[KS91a] I. Karatzas and S. E. Shreve, Brownian motion and stochastic calculus, 2nd ed., Graduate Texts in Mathematics, vol. 113, Springer-Verlag, New York, 1991. MR 1121940

[KS91b] J. Krug and H. Spohn, Kinetic roughening of growing surfaces, in Solids Far From Equilibrium: Growth, Morphology and Defects (C. Godreche, ed.), Cambridge University Press, Cambridge, 1991.

[KS12] S. Kuksin and A. Shirikyan, Mathematics of two-dimensional turbulence, Cambridge Tracts in Mathematics, vol. 194, Cambridge University Press, Cambridge, 2012. MR.3443633

[KT73] J. M. Kosterlitz and D. J. Thouless, Ordering, metastability and phase transitions in two-dimensional systems, J. Physics C: Solid State Physics, 6 (1973), no. 7, 1181.

[Kup10] A. Kupiainen, Ergodicity of two dimensional turbulence, arXiv:1005.0587, 2010.

[Kup16] A. Kupiainen, Renormalization group and stochastic PDEs, Ann. Henri Poincaré 17 (2016), no. 3, 497-535, DOI 10.1007/s00023-015-0408-y. MR3459120

[Lab17] C. Labbé, Weakly asymmetric bridges and the KPZ equation, Comm. Math. Phys. 353 (2017), no. 3, 1261-1298, DOI 10.1007/s00220-017-2875-0. MR3652491

[LR15] W. Liu and M. Röckner, Stochastic partial differential equations: an introduction, Universitext, Springer, Cham, 2015. MR3410409

[Lyo98] T. J. Lyons, Differential equations driven by rough signals, Rev. Mat. Iberoamericana 14 (1998), no. 2, 215-310, DOI 10.4171/RMI/240. MR 1654527

[Mat03] J. C. Mattingly, On recent progress for the stochastic Navier Stokes equations, Journées "Équations aux Dérivées Partielles", Univ. Nantes, Nantes, 2003, pp. Exp. No. XI, 52. MR2050597

[Mat18] K. Matetski, Martingale-driven approximations of singular stochastic PDEs, arXiv: 1808.09429, 2018.

[McK95] H. P. McKean, Statistical mechanics of nonlinear wave equations. IV. Cubic Schrödinger, Comm. Math. Phys. 168 (1995), no. 3, 479-491. MR1328250

[MP17] J. Martin and N. Perkowski, Paracontrolled distributions on Bravais lattices and weak universality of the 2d parabolic anderson model, arXiv:1704.08653, 2017.

[MS77] O. A. McBryan and T. Spencer, On the decay of correlations in $\mathrm{SO}(n)$-symmetric ferromagnets, Comm. Math. Phys. 53 (1977), no. 3, 299-302. MR0441179

[MU18] J. Magnen and J. Unterberger, The scaling limit of the KPZ equation in space dimension 3 and higher, J. Stat. Phys. 171 (2018), no. 4, 543-598, DOI 10.1007/s10955018-2014-0. MR3790153

[Mue91] C. Mueller, On the support of solutions to the heat equation with noise, Stochastics Stochastics Rep. 37 (1991), no. 4, 225-245, DOI 10.1080/17442509108833738. MR.1149348

[MW17a] J.-C. Mourrat and H. Weber, Convergence of the two-dimensional dynamic IsingKac model to $\Phi_{2}^{4}$, Comm. Pure Appl. Math. 70 (2017), no. 4, 717-812, DOI 10.1002/cpa.21655. MR.3628883

[MW17b] J.-C. Mourrat and H. Weber, The dynamic $\Phi_{3}^{4}$ model comes down from infinity, Comm. Math. Phys. 356 (2017), no. 3, 673-753, DOI 10.1007/s00220-017-2997-4. MR.3719541

[MW18] A. Moinat and H. Weber, Space-time localisation for the dynamic $\phi_{3}^{4}$ model, arXiv: $1811.05764,2018$.

[OR98] M. Oberguggenberger and F. Russo, Nonlinear stochastic wave equations, Integral Transform. Spec. Funct. 6 (1998), no. 1-4, 71-83, DOI 10.1080/10652469808819152. Generalized functions - linear and nonlinear problems (Novi Sad, 1996). MR 1640497

[OW18] F. Otto and H. Weber, Quasilinear SPDEs via Rough Paths, Arch. Ration. Mech. Anal. 232 (2019), no. 2, 873-950, DOI 10.1007/s00205-018-01335-8. MR3925533

[Par17] S. Parekh, The KPZ limit of ASEP with boundary, Comm. Math. Phys. 365 (2019), no. 2, 569-649, DOI 10.1007/s00220-018-3258-x. MR3907953 
[Pau35] L. Pauling, The structure and entropy of ice and of other crystals with some randomness of atomic arrangement, J. Amer. Chem. Soc., 57 (1935), no. 12, 2680-2684.

[PR07] C. Prévôt and M. Röckner, A concise course on stochastic partial differential equations, Lecture Notes in Mathematics, vol. 1905, Springer, Berlin, 2007. MR2329435

[PR18] N. Perkowski and T. C. Rosati, The KPZ equation on the real line, arXiv:1808.00354, 2018.

[PW81] G. Parisi and Y. S. Wu, Perturbation theory without gauge fixing, Sci. Sinica 24 (1981), no. 4, 483-496. MR626795

[RWZZ18] M. Röckner, B. Wu, R. Zhu, and X. Zhu, Stochastic heat equations with values in a Riemannian manifold, Atti Accad. Naz. Lincei Rend. Lincei Mat. Appl. 29 (2018), no. 1, 205-213, DOI 10.4171/RLM/801. MR.3787728

[She07] S. Sheffield, Gaussian free fields for mathematicians, Probab. Theory Related Fields 139 (2007), no. 3-4, 521-541, DOI 10.1007/s00440-006-0050-1. MR2322706

[She18] H. Shen, Stochastic quantization of an Abelian gauge theory, arXiv:1801.04596, 2018.

[Spo86] H. Spohn, Equilibrium fluctuations for interacting Brownian particles, Comm. Math. Phys. 103 (1986), no. 1, 1-33. MR826856

[Spo14] H. Spohn, Nonlinear fluctuating hydrodynamics for anharmonic chains, J. Stat. Phys. 154 (2014), no. 5, 1191-1227, DOI 10.1007/s10955-014-0933-y. MR.3176405

[ST18] H. Shen and L.-C. Tsai, Stochastic telegraph equation limit for the stochastic six vertex model, arXiv:1807.04678, 2018.

[SW18] H. Shen and H. Weber, Glauber dynamics of 2D Kac-Blume-Capel model and their stochastic PDE limits, J. Funct. Anal. 275 (2018), no. 6, 1321-1367, DOI 10.1016/j.jfa.2017.12.014. MR3820327

[SX16] H. Shen and W. Xu, Weak universality of dynamical $\Phi_{3}^{4}$ : non-Gaussian noise, Stoch. Partial Differ. Equ. Anal. Comput. 6 (2018), no. 2, 211-254, DOI 10.1007/s40072017-0107-4. MR3818405

[Wal86] J. B. Walsh, An introduction to stochastic partial differential equations, École d'été de probabilités de Saint-Flour, XIV-1984, Lecture Notes in Math., vol. 1180, Springer, Berlin, 1986, pp. 265-439, DOI 10.1007/BFb0074920. MR876085

[Yan18] K. Yang, The KPZ equation, non-equilibrium solutions, and weak universality for long-range interactions, arXiv:1810.02836, 2018.

[Zhu90] M. Zhu, Equilibrium fluctuations for one-dimensional Ginzburg-Landau lattice model, Nagoya Math. J. 117 (1990), 63-92, DOI 10.1017/S0027763000001811. MR.1044937

[ZZ15] R. Zhu and X. Zhu, Three-dimensional Navier-Stokes equations driven by spacetime white noise, J. Differential Equations 259 (2015), no. 9, 4443-4508, DOI 10.1016/j.jde.2015.06.002. Note that the most recent version arXiv:1406.0047 fixed a mistake in the journal version of this paper. MR.3373412

[ZZ18] R. Zhu and X. Zhu, Lattice approximation to the dynamical $\Phi_{3}^{4}$ model, Ann. Probab. 46 (2018), no. 1, 397-455, DOI 10.1214/17-AOP1188. MR.3758734

Department of Mathematics, Columbia University, 2990 Broadway, New York, New YORK 10027

Email address: corwin@math.columbia.edu

Department of Mathematics, University of Wisconsin-Madison, 480 Lincoln Drive, MAdison, Wisconsin 53706

Email address: pkushenhao@gmail.com 